\title{
Multipolar effective-one-body waveforms for precessing binary black holes: Construction and validation
}

\author{
Serguei Ossokine $\odot,{ }^{1}$ Alessandra Buonanno, ${ }^{1,2}$ Sylvain Marsat, ${ }^{3,1}$ Roberto Cotesta $\odot,{ }^{1}$ Stanislav Babak ${ }^{3,1,4}$ Tim Dietrich, ${ }^{5,1}$ \\ Roland Haas $\odot,{ }^{6,1}$ Ian Hinder $\odot,{ }^{7,1}$ Harald P. Pfeiffer, ${ }^{1}$ Michael Pürrer $\odot,{ }^{1}$ Charles J. Woodford $\odot,{ }^{8,9}$ Michael Boyle, ${ }^{10}$ \\ Lawrence E. Kidder $\odot{ }^{10}$ Mark A. Scheel, ${ }^{11}$ and Béla Szilágyi® ${ }^{12,11}$ \\ ${ }^{1}$ Max Planck Institute for Gravitational Physics (Albert Einstein Institute), \\ Am Mühlenberg 1, Potsdam 14476, Germany \\ ${ }^{2}$ Department of Physics, University of Maryland, College Park, Maryland 20742, USA \\ ${ }^{3}$ APC, Univ Paris Diderot, CNRS/IN2P3, CEA/Irfu, Obs de Paris, Sorbonne Paris Cité, France \\ ${ }^{4}$ Moscow Institute of Physics and Technology, Dolgoprudny, Moscow region, Russia \\ ${ }^{5}$ Institute for Physics and Astronomy, University of Potsdam, \\ Karl-Liebknecht-Str. 24/25, 14476 Potsdam, Germany \\ ${ }^{6}$ NCSA, University of Illinois at Urbana-Champaign, Urbana, Illinois 61801, USA \\ ${ }^{7}$ University of Manchester, Sackville Building, Granby Row, Manchester M1 3BU, United Kingdom \\ ${ }^{8}$ Department of Physics 60 St. George Street, University of Toronto, Toronto, Ontario M5S 3H8, Canada \\ ${ }^{9}$ Canadian Institute for Theoretical Astrophysics, 60 St. George Street, University of Toronto, \\ Toronto, Ontario M5S 3H8, Canada \\ ${ }^{10}$ Cornell Center for Astrophysics and Planetary Science, Cornell University, \\ Ithaca, New York 14853, USA \\ ${ }^{11}$ Theoretical Astrophysics 350-17, California Institute of Technology, Pasadena, California 91125, USA \\ ${ }^{12}$ Jet Propulsion Laboratory, California Institute of Technology, Pasadena, California 91109, USA
}

(Received 29 April 2020; accepted 7 July 2020; published 31 August 2020)

\begin{abstract}
As gravitational-wave detectors become more sensitive and broaden their frequency bandwidth, we will access a greater variety of signals emitted by compact binary systems, shedding light on their astrophysical origin and environment. A key physical effect that can distinguish among different formation scenarios is the misalignment of the spins with the orbital angular momentum, causing the spins and the binary's orbital plane to precess. To accurately model such precessing signals, especially when masses and spins vary in the wide astrophysical range, it is crucial to include multipoles beyond the dominant quadrupole. Here, we develop the first multipolar precessing waveform model in the effective-one-body (EOB) formalism for the entire coalescence stage (i.e., inspiral, merger and ringdown) of binary black holes: SEOBNRV4PHM. In the nonprecessing limit, the model reduces to SEOBNRV4HM, which was calibrated to numerical-relativity (NR) simulations, and waveforms from black-hole perturbation theory. We validate SEOBNRV4PHM by comparing it to the public catalog of 1405 precessing NR waveforms of the Simulating eXtreme Spacetimes (SXS) collaboration, and also to 118 SXS precessing NR waveforms, produced as part of this project, which span mass ratios 1-4 and (dimensionless) black-hole's spins up to 0.9. We stress that SEOBNRV4 PHM is not calibrated to NR simulations in the precessing sector. We compute the unfaithfulness against the $1523 \mathrm{SXS}$ precessing NR waveforms, and find that, for $94 \%(57 \%)$ of the cases, the maximum value, in the total mass range $20-200 M_{\odot}$, is below $3 \%(1 \%)$. Those numbers change to $83 \%$ (20\%) when using the inspiral-merger-ringdown, multipolar, precessing phenomenological model IMRPhenomPv3HM. We investigate the impact of such unfaithfulness values with two Bayesian, parameter-estimation studies on synthetic signals. We also compute the unfaithfulness between those waveform models as a function of the mass and spin parameters to identify in which part of the parameter space they differ the most. We validate them also against the multipolar, precessing NR surrogate model NRSur7dq4, and find that the SEOBNRv4PHM model outperforms IMRPhenomPv3HM.
\end{abstract}

DOI: 10.1103/PhysRevD.102.044055

Published by the American Physical Society under the terms of the Creative Commons Attribution 4.0 International license. Further distribution of this work must maintain attribution to the author(s) and the published article's title, journal citation, and DOI. Open access publication funded by the Max Planck Society.

\section{INTRODUCTION}

Since the Laser Interferometer Gravitational wave Observatory (LIGO) detected a gravitational wave (GWs) from a binary-black-hole (BBH) in 2015 [1], multiple observations of GWs from BBHs have been made with 
the LIGO [2] and Virgo [3] detectors [4-9]. Two binary neutron star (BNSs) systems have been observed [10,11], one of them both in gravitational and electromagnetic radiation [12,13], opening the exciting new chapter of multimessenger GW astronomy. Mergers of compact-object binaries are expected to be detected at an even higher rate with LIGO and Virgo ongoing and future, observing runs [14], and with subsequent third-generation detectors on the ground, such as the Einstein Telescope and Cosmic Explorer, and the Laser Interferometer Space Antenna (LISA). In order to extract the maximum amount of astrophysical and cosmological information, the accurate modeling of GWs from binary systems is more critical than ever. Great progress has been made in this direction, both through the development of analytical methods to solve the two-body problem in general relativity (GR), and by ever more expansive numerical-relativity (NR) simulations.

One of the key areas of interest is to improve the modeling of systems where the misalignment of the spins with the orbital angular momentum causes the spins and the orbital plane to precess [15]. Moreover, when the binary's component masses are asymmetric, gravitational radiation is no longer dominated by the quadrupole moment, and higher multipoles need to be accurately modeled [16]. Precession and higher multipoles lead to very rich dynamics, which in turn is imprinted on the GW signal (see, e.g., $[15,17-31])$. Their measurements will be able to shed light on the formation mechanism of the observed systems, probe the astrophysical environment, break degeneracy among parameters, allowing more accurate measurements of cosmological parameters, masses and spins, and more sophisticated tests of GR.

Faithful waveform models for precessing compactobject binaries have been developed within the effectiveone-body (EOB) formalism [26,32,33], and the phenomenological approach [34-38] through calibration to NR simulations. Recently, an inspiral-merger-ringdown phenomenological waveform model that tracks precession and includes higher modes was constructed in Ref. [39] (henceforth, IMRPhenomPv $3 \mathrm{HM})^{1}$ The model describes the six spin degrees of freedom in the inspiral phase, but not in the late-inspiral, merger and ringdown stages. In the coprecessing frame $[21,23,42-44]$, in which the BBH is viewed faceon at all times and the GW radiation resembles the nonprecessing one, it includes the modes $(\ell, m)=(2, \pm 2)$, $(2, \pm 1),(3, \pm 3),(3, \pm 2),(4, \pm 4)$ and $(4, \pm 3)$. Here, building on the multipolar aligned-spin EOB waveform model of Ref. [45,46], which was calibrated to 157 NR simulations $[47,48]$, and 13 waveforms from $\mathrm{BH}$ perturbation theory for

\footnotetext{
${ }^{1}$ During the final preparation of this work, a new frequencydomain phenomenological model with precession and higher modes (IMRPhenomXPHM [40]), and a time-domain phenomenological precessing model with the dominant mode (IMRPhenomTP [41]) were developed. We leave the comparison to these models for future work.
}

the (plunge-)merger and ringdown [49], we develop the first EOB waveform model that includes both spinprecession and higher modes (henceforth, SEOBNRV4 PHM) . The model describes the six spin degrees of freedom throughout the $\mathrm{BBH}$ coalescence. It differs from the one of Refs. [26,33], not only because it includes in the coprecessing frame the $(3, \pm 3),(4, \pm 4)$ and $(5, \pm 5)$ modes, beyond the $(2, \pm 2)$ and $(2, \pm 1)$ modes, but also because it uses an improved description of the two-body dynamics, having been calibrated [45] to a large set of NR waveforms [47]. We note that IMRPhenomPv3HM and SEOBNRV4 PHM are not completely independent because the former is constructed fitting (in frequency domain) hybridized waveforms obtained by stitching together EOB and NR waveforms. We stress that both SEOBNRV4HM and IMRPhenomPv3 HM are not calibrated to NR simulations in the precessing sector. Finally, the surrogate approach, which interpolates NR waveforms, has been used to construct several waveform models that include higher modes [50] and precession [51]. In this paper, we consider the state-ofthe-art surrogate waveform model with full spin precession and higher modes [52] (henceforth, NRSur7dq4), developed for binaries with mass ratios 1-4, (dimensionless) BH's spins up to 0.8 and binary's total masses larger than $\sim 60 M_{\odot}$. It includes in the coprecessing frame all modes up to $\ell=4$. Table I summarizes the waveform models used in this paper.

The best tool at our disposal to validate waveform models built from approximate solutions of the Einstein equations, such as the ones obtained from post-Newtonian (PN) theory, BH perturbation theory and the EOB approach, is their comparison to NR waveforms. So far, NR simulations of BBHs have been mostly limited to mass ratio $\leq 4$ and (dimensionless) spins $\leq 0.8$, and length of 15-20 orbital cycles before merger [53-57] (however, see Ref. [58] where simulations with larger spins and mass ratios were obtained through a synergistic use of NR codes). Here, to test our newly constructed EOB precessing waveform model, we enhance the NR parameter-space coverage, while maintaining a manageable computational

TABLE I. The waveform models used in this paper. We also specify which modes are included in the coprecessing frame.

\begin{tabular}{lll}
\hline \hline Model name & Modes in the coprecessing frame & Reference \\
\hline SEOBNRv3P & $(2, \pm 2),(2, \pm 1)$ & {$[26,33]$} \\
SEOBNRv4P & $(2, \pm 2),(2, \pm 1)$ & this work \\
SEOBNRv4PHM & $(2, \pm 2),(2, \pm 1),(3, \pm 3),(4, \pm 4)$ & \\
& $(5, \pm 5)$ & this work \\
IMRPhenomPv2 & $(2, \pm 2)$ & {$[34]$} \\
IMRPhenomPv3 & $(2, \pm 2)$ & {$[36]$} \\
IMRPhenomPv3HM & $(2, \pm 2),(2, \pm 1),(3, \pm 3),(3, \pm 2)$, & \\
& $(4, \pm 4),(4, \pm 3)$ & {$[39]$} \\
NRSur7dq4 & all with $\ell \leq 4$ & {$[52]$} \\
\hline \hline
\end{tabular}


cost, and perform 118 new NR simulations with the pseudo spectral Einstein code (SpEC) of the Simulating eXtreme Spacetimes (SXS) collaboration. The new NR simulations span BBHs with mass ratios 1-4, and dimensionless spins in the range $0.3-0.9$, and different spins' orientations. To assess the accuracy of the different precessing waveform models, we compare them to the NR waveforms of the public SXS catalogue [57], and to the new $118 \mathrm{NR}$ waveforms produced for this paper.

The paper is organized as follows. In Sec. II we discuss the new NR simulations of BBHs, and assess their numerical error. In Sec. III we develop the multipolar EOB waveform model for spin-precessing BBHs, SEOBNRv4PHM, and highlight the improvements with respect to the previous version [26,33], which was used in LIGO and Virgo inference analyses $[5,59,60]$. In Sec. IV we validate the accuracy of the multipolar precessing EOB model by comparing it to NR waveforms. We also compare the performance of SEOBNRV4PHM against the one of IMRPhenomPV3HM, and study in which region of the parameter space those models differ the most from NR simulations, and also from each other. In Sec. V we use Bayesian analysis to explore the impact of the accuracy of the precessing waveform models when extracting astrophysical information and perform two synthetic NR injections in zero noise. In Sec. VI we summarize our main conclusions and discuss future work. Finally, in Appendix A we compare the precessing waveform models to the NR surrogate NRSur7dq4, and in Table II (in Appendix B) we list the parameters of the $118 \mathrm{NR}$ simulations done for this paper.

In what follows, we use geometric units $G=1=c$ unless otherwise specified.

\section{NEW NUMERICAL RELATIVITY SIMULATIONS OF SPINNING, PRECESSING BINARY BLACK HOLES}

Henceforth, we denote with $m_{1,2}$ the two BH masses (with $m_{1} \geq m_{2}$ ), $\boldsymbol{S}_{1,2} \equiv m_{1,2}^{2} \chi_{1,2}$ their spins, $q=m_{1} / m_{2}$ the mass ratio, $M=m_{1}+m_{2}$ the binary's total mass, $\mu=$ $m_{1} m_{2} / M$ the reduced mass, and $\nu=\mu / M$ the symmetric mass ratio. We indicate with $\boldsymbol{J}=\boldsymbol{L}+\boldsymbol{S}$ the total angular momentum, where $\boldsymbol{L}$ and $\boldsymbol{S}=\boldsymbol{S}_{1}+\boldsymbol{S}_{2}$, are the orbital angular momentum and the total spin, respectively.

\section{A. New 118 precessing numerical-relativity waveforms}

The spectral Einstein code (SPEC) ${ }^{2}$ of the Simulating eXtreme Spacetimes (SXS) collaboration is a multi-domain collocation code designed for the solution of partial differential equations on domains with simple topologies. It has been used extensively to study the mergers of

\footnotetext{
${ }^{2}$ www.black-holes.org
}

compact-object binaries composed of $\mathrm{BH}[31,57,61-64]$ and NSs [65-68], including in theories beyond GR [6972]. SPEC employs a first-order symmetric-hyperbolic formulation of Einstein's equations [73] in the damped harmonic gauge $[74,75]$. Dynamically controlled excision boundaries are used to treat spacetime singularities $[61,76]$ (see Ref. [57] for a recent, detailed overview).

Significant progress has been made in recent years by several NR groups to improve the coverage of the BBH parameter space [53-58], mainly motivated by the calibration of analytical waveform models and surrogate models used in LIGO and Virgo data analysis. While large strides have been made for aligned-spin cases, the exploration of precessing waveforms has been mostly limited to $q \leq 4, \chi_{1,2} \equiv\left|\chi_{1,2}\right| \leq 0.8$, typically $15-20$ orbital cycles before merger, and a large region of parameter space remains to be explored. Simulations with high mass ratio $(q \geq 4)$ and high spin $\left(\left|\chi_{1}\right|>0.5\right)$ are challenging, primarily due to the need to resolve the disparate length scales in the binary system, which increases the computational cost for a given level of accuracy. Furthermore, for high spin, the apparent horizons can be dramatically smaller, which makes it more difficult to control the excision boundaries, further increasing the computational burden.

Here, we want to improve the parameter-space coverage of the SXS catalog [57], while maintaining a manageable computational cost, thus we restrict to simulations in the range of mass ratios $q=1-4$ and (dimensionless) spins $\chi_{1,2}=0.3-0.9$, with the spin magnitudes decreasing as the mass ratio increases. In Fig. 1 we display, in the $q-\chi_{1}$ parameter space, the precessing and non-precessing waveforms from the published SXS catalogue [57], and the new precessing waveforms produced as part of this work.

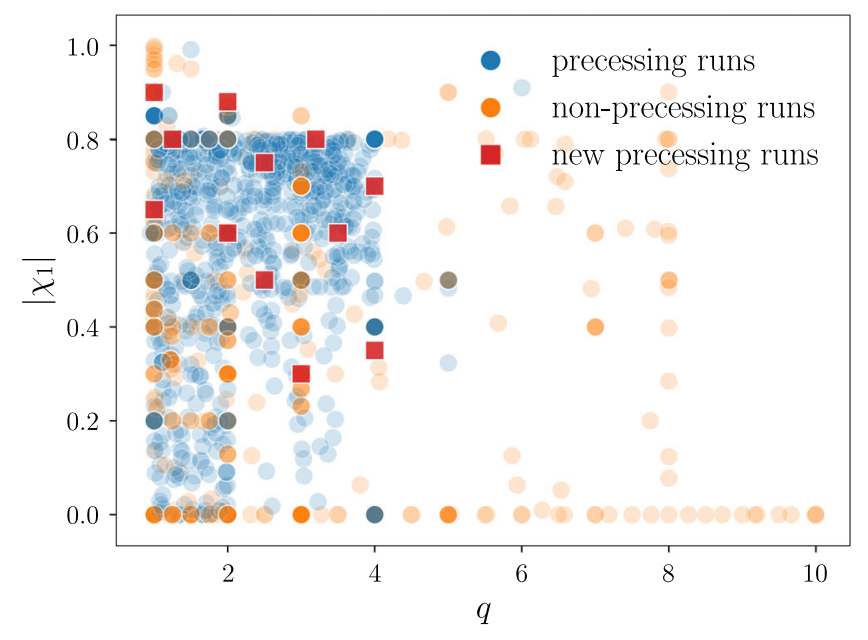

FIG. 1. Parameter space coverage in $q-\chi_{1}$ space for SpEC waveforms. For runs from the SpEC catalog [57] the opacity was changed so that runs with similar parameters are clearly visible. We indicate with squares precessing $\mathrm{BBH}$ runs performed as part of this paper. 
We choose to start all the simulations at the same (angular) orbital frequency, $M \Omega_{0} \approx 0.0157$, where the value is not exact as it was modified slightly during the eccentricity-reduction procedure in SPEC [77]. This corresponds to a physical GW starting frequency of $20 \mathrm{~Hz}$ at $50 M_{\odot}$ and results in the number of orbits up to merger varying between 15 and 30 in our new catalog.

We parametrize the directions of the spins by three angles, the angles $\theta_{1,2}$ between the spins and the unit vector along the Newtonian orbital angular momentum, $\hat{\boldsymbol{L}}_{\mathrm{N}}$, and the angle $\Delta \phi$ between the projections of the spins in the orbital plane. Explicitly,

$$
\begin{aligned}
\theta_{i} & =\arccos \left(\chi_{i} \cdot \hat{\boldsymbol{L}}_{\mathrm{N}}\right), \\
\Delta \phi & =\arccos \left(\frac{\cos \theta_{12}-\cos \theta_{1} \cos \theta_{2}}{\sin \theta_{1} \sin \theta_{2}}\right),
\end{aligned}
$$

where $\cos \theta_{12}=\chi_{1} \cdot \chi_{2}$. We make the choice that $\chi_{1}$ lies in the $\hat{\boldsymbol{L}}_{\mathrm{N}}-\mathbf{n}$ plane, where $\mathbf{n}$ is the unit vector along the binary's radial separation, at the start of the simulation. The angles are chosen to be $\theta_{i, 0} \in\left\{60^{\circ}, \theta_{\max }\right\}$, and $\Delta \phi_{0} \in\left\{0,90^{\circ}\right\}$. Here $\theta_{\max }$ is the angle that maximizes the opening angle of $\boldsymbol{L}_{\mathrm{N}}$ around the total angular momentum $\boldsymbol{J}$ and is computed assuming that the two spins are colinear, giving

$$
\cos \theta_{\text {max }}=-\frac{|\mathbf{S}|}{\left|\boldsymbol{L}_{\mathrm{N}}\right|}=-\frac{m_{1}^{2} \chi_{1}+m_{2}^{2} \chi_{2}}{\left|\boldsymbol{L}_{\mathrm{N}}\right|},
$$

with $\left|\boldsymbol{L}_{\mathrm{N}}\right|=\mu M^{2 / 3} \Omega^{-1 / 3}$ for circular orbit, being $\Omega$ the orbital angular frequency. For each choice of $\{q, \chi\}$ we choose 10 different configurations divided into two categories: (i) $\chi_{1}=\chi_{2}=\chi, \theta_{i, 0} \in\left\{60^{\circ}, \theta_{\max }\right\}, \Delta \phi_{0} \in\left\{0,90^{\circ}\right\}$ giving eight runs, and (ii) $\chi_{1}=\chi, \chi_{2}=0, \theta_{1,0} \in\left\{60^{\circ}, \theta_{\max }\right\}$ giving two runs. The detailed parameters can be found in Appendix B.

These choices of the spin directions allow us to test the multipolar precessing model SEOBNRV4PHM in many different regimes, including where the effects of precession are maximized, and where spin-spin effects are significant or diminished.

\section{B. Unfaithfulness for spinning, precessing waveforms}

The gravitational signal emitted by noneccentric BBH systems and observed by a detector depends on 15 parameters: the component masses $m_{1}$ and $m_{2}$ (or equivalently the mass ratio $q=m_{1} / m_{2} \geq 1$ and the total mass $\left.M=m_{1}+m_{2}\right)$, the dimensionless spins $\boldsymbol{\chi}_{1}(t)$ and $\boldsymbol{\chi}_{2}(t)$, the direction to observer from the source described by the angles $\left(l, \varphi_{0}\right)$, the luminosity distance $d_{L}$, the polarization $\psi$, the location in the sky $(\theta, \phi)$ and the time of arrival $t_{c}$. The gravitational strain can be written as:

$$
\begin{aligned}
h(t) \equiv & F_{+}(\theta, \phi, \psi) h_{+}\left(l, \varphi_{0}, d_{L}, \xi, t_{\mathrm{c}} ; t\right) \\
& +F_{\times}(\theta, \phi, \psi) h_{\times}\left(l, \varphi_{0}, d_{L}, \xi, t_{\mathrm{c}} ; t\right),
\end{aligned}
$$

where to simplify the notation we introduce the function $\xi \equiv\left(q, M, \chi_{1}(t), \chi_{2}(t)\right)$. The functions $F_{+}(\theta, \phi, \psi)$ and $F_{\times}(\theta, \phi, \psi)$ are the antenna patterns [78,79]:

$$
\begin{aligned}
F_{+}(\theta, \phi, \psi)= & \frac{1+\cos ^{2}(\theta)}{2} \cos (2 \phi) \cos (2 \psi) \\
& -\cos (\theta) \sin (2 \phi) \sin (2 \psi), \\
F_{\times}(\theta, \phi, \psi)= & \frac{1+\cos ^{2}(\theta)}{2} \cos (2 \phi) \sin (2 \psi) \\
& +\cos (\theta) \sin (2 \phi) \cos (2 \psi) .
\end{aligned}
$$

Equation (2.3) can be rewritten as:

$$
\begin{aligned}
h(t) \equiv & \mathcal{A}(\theta, \phi)\left[\cos \kappa(\theta, \phi, \psi) h_{+}\left(l, \varphi_{0}, d_{L}, \boldsymbol{\xi}, t_{c} ; t\right)\right. \\
& \left.+\sin \kappa(\theta, \phi, \psi) h_{\times}\left(l, \varphi_{0}, d_{L}, \boldsymbol{\xi}, t_{c} ; t\right)\right],
\end{aligned}
$$

where $\kappa(\theta, \phi, \psi)$ is the effective polarization [80] defined as:

$$
e^{i \kappa(\theta, \phi, \psi)}=\frac{F_{+}(\theta, \phi, \psi)+i F_{\times}(\theta, \phi, \psi)}{\sqrt{F_{+}^{2}(\theta, \phi, \psi)+F_{\times}^{2}(\theta, \phi, \psi)}},
$$

which has support in the region $[0,2 \pi)$, while $\mathcal{A}(\theta, \phi)$ reads:

$$
\mathcal{A}(\theta, \phi)=\sqrt{F_{+}^{2}(\theta, \phi, \psi)+F_{\times}^{2}(\theta, \phi, \psi)} .
$$

Henceforth, to ease the notation we suppress the dependence on $(\theta, \phi, \psi)$ in $\kappa$.

Let us introduce the inner product between two waveforms $a$ and $b[78,79]$ :

$$
(a, b) \equiv 4 \operatorname{Re} \int_{f_{\text {in }}}^{f_{\max }} d f \frac{\tilde{a}(f) \tilde{b}^{*}(f)}{S_{n}(f)}
$$

where a tilde indicates the Fourier transform, a star the complex conjugate and $S_{n}(f)$ is the one-sided power spectral density (PSD) of the detector noise. We employ as PSD the Advanced LIGO's "zero-detuned high-power" design sensitivity curve [81]. Here we use $f_{\text {in }}=10 \mathrm{~Hz}$ and $f_{\max }=2 \mathrm{kHz}$, when both waveforms fill the band. For cases where this is not the case (e.g., the NR waveforms) we set $f_{\text {in }}=1.05 f_{\text {start }}$, where $f_{\text {start }}$ is the starting frequency of the waveform.

To assess the closeness between two waveforms $s$ (e.g., the signal) and $\tau$ (e.g., the template), as observed by a detector, we define the following faithfulness function [46]: 


$$
\mathcal{F}\left(M_{s}, l_{s}, \varphi_{0 s}, \kappa_{s}\right) \equiv \max _{t_{c}, \varphi_{0 \tau}, \kappa_{\tau}}\left[\left.\frac{(s, \tau)}{\sqrt{(s, s)(\tau, \tau)}}\right|_{\substack{\xi_{s}\left(t_{s}=t_{0_{s}}\right)=\xi_{\tau}\left(t_{\tau}=t_{0} \tau \\ l_{\tau}=l_{\tau}\right)}}\right] .
$$

While in the equation above we set the inclination angle $r$ of signal and template waveforms to be the same, the coalescence time $t_{c}$ and the angles $\varphi_{0 \tau}$ and $\kappa_{\tau}$ of the template waveform are adjusted to maximize the faithfulness. This is a typical choice motivated by the fact these quantities are not interesting from an astrophysical perspective. The maximizations over $t_{c}$ and $\varphi_{0 \tau}$ are performed numerically, while the maximization over $\kappa_{\tau}$ is done analytically following the procedure described in Ref. [80] (see Appendix A therein).

The condition $\boldsymbol{\xi}_{\mathrm{s}}\left(t_{\mathrm{s}}=t_{0_{\mathrm{s}}}\right)=\boldsymbol{\xi}_{\tau}\left(t_{\tau}=t_{0_{\tau}}\right)$ in Eq. (2.9) enforces that the mass ratio $q$, the total mass $M$ and the spins $\chi_{1,2}$ of the template waveform at $t=t_{0}$ (i.e., at the beginning of the waveform) are set to have the same values of the ones in the signal waveform at its $t_{0}$. When computing the faithfulness between NR waveforms with different resolutions this condition is trivially satisfied by the fact that they are generated using the same initial data. In the case of the faithfulness between NR and any model from the SEOBNR family, it is first required to ensure that $t_{0}$ has the same physical meaning for both waveforms. Ideally $t=t_{0_{\tau}}$ in the SEOBNRwaveform should be fixed by requesting that the frequency of the SEOBNR $(2,2)$ mode at $t_{0_{\tau}}$ coincides with the NR $(2,2)$ mode frequency at $t_{0_{d}}$. This is in practice not possible because the NR $(2,2)$ mode frequency may display small oscillations caused by different effects-for example the persistence of the junk radiation, some residual orbital eccentricity or spin-spin couplings [77]. Thus, the frequency of the SEOBNR $(2,2)$ mode at $t=t_{0_{\tau}}$ is chosen to guarantee the same time-domain length of the NR waveform. ${ }^{3}$ In practice, we require that the peak of $\sum_{\ell, m}\left|h_{\ell m}\right|^{2}$, as elapsed respectively from $t_{0_{s}}$ and $t_{0_{\tau}}$, occurs at the same time in NR and SEOBNR. For waveforms from the IMRPhenom family we adopt a different approach, and following the method outlined in Ref. [36], also optimize the faithfulness numerically over the reference frequency of the waveform.

The faithfulness defined in Eq. (2.9) is still a function of 4 parameters (i.e., $M_{s}, l_{s}, \varphi_{0 s}, \kappa_{s}$ ), therefore it does not allow to describe the agreement between waveforms in a compact form. For this purpose we define the sky-andpolarization-averaged faithfulness [33] as:

$\overline{\mathcal{F}}\left(M_{s}, l_{s}\right) \equiv \frac{1}{8 \pi^{2}} \int_{0}^{2 \pi} d \kappa_{s} \int_{0}^{2 \pi} d \varphi_{0 s} \mathcal{F}\left(M_{s}, l_{s}, \varphi_{0 s}, \kappa_{s}\right)$.

Despite the apparent difference, the sky-and-polarizationaveraged faithfulness $\overline{\mathcal{F}}$ defined above is equivalent to the one given in Eqs. (9) and (B15) of Ref. [33]. The definition in Eq. (2.10) is less computationally expensive because, thanks to the parametrization of the waveforms in Eq. (2.5), it allows one to write the sky-and-polarization-averaged faithfulness as a double integral instead of the triple integral in Eq. (B15) of Ref. [33]. We also define the sky-and-polarizationaveraged, signal-to-noise (SNR)-weighted faithfulness as:

$$
\overline{\mathcal{F}}_{\mathrm{SNR}}\left(M_{s}, l_{s}\right) \equiv \sqrt[3]{\frac{\int_{0}^{2 \pi} d \kappa_{s} \int_{0}^{2 \pi} d \varphi_{0 s} \mathcal{F}^{3}\left(M_{s}, l_{s}, \varphi_{0 s}, \kappa_{s}\right) \mathrm{SNR}^{3}\left(l_{s}, \varphi_{0 s}, \kappa_{s}\right)}{\int_{0}^{2 \pi} d \kappa_{s} \int_{0}^{2 \pi} d \varphi_{0 s} \mathrm{SNR}^{3}\left(l_{s}, \varphi_{0 s}, \kappa_{s}\right)}}
$$

where the $\operatorname{SNR}\left(l_{s}, \varphi_{0 s}, \theta_{s}, \phi_{s}, \kappa_{s}, D_{\mathrm{L}_{s}}, \boldsymbol{\xi}_{s}, t_{c_{s}}\right)$ is defined as:

$\operatorname{SNR}\left(l_{s}, \varphi_{0 s}, \theta_{s}, \phi_{s}, \kappa_{s}, D_{\mathrm{L}_{s}}, \boldsymbol{\xi}_{s}, t_{c_{s}}\right) \equiv \sqrt{\left(h_{s}, h_{s}\right)}$.

This is also an interesting metric because weighting the faithfulness with the SNR takes into account that, at fixed distance, the SNR of the signal depends on its phase and on the effective polarization (i.e., a combination of waveform polarization and sky-position). Since the SNR scales with the luminosity distance, the number of detectable sources scale with the $\mathrm{SNR}^{3}$, therefore signals with a smaller SNR

\footnotetext{
${ }^{3}$ The difference between the NR $(2,2)$ mode frequency and the SEOBNRV4 PHM $(2,2)$ frequency chosen at $t=t_{0}$ is never larger than $5 \%$.
}

are less likely to be observed. Finally, we define the unfaithfulness (or mismatch) as

$$
\overline{\mathcal{M}}=1-\overline{\mathcal{F}}
$$

\section{Accuracy of new numerical-relativity waveforms}

To assess the accuracy of the new NR waveforms, we compute the sky-and-polarization-averaged unfaithfulness defined in Eq. (2.10) between the highest and second highest resolutions in the NR simulation.

Figure 2 shows a histogram of the unfaithfulness, evaluated at $l_{s}=\pi / 3$ maximized over the total mass, between 20 and $200 M_{\odot}$. It is apparent that the unfaithfulness is below $1 \%$ for most cases, but there are several cases with much higher unfaithfulness. This tail to high unfaithfulness has been observed previously, when evaluating the 


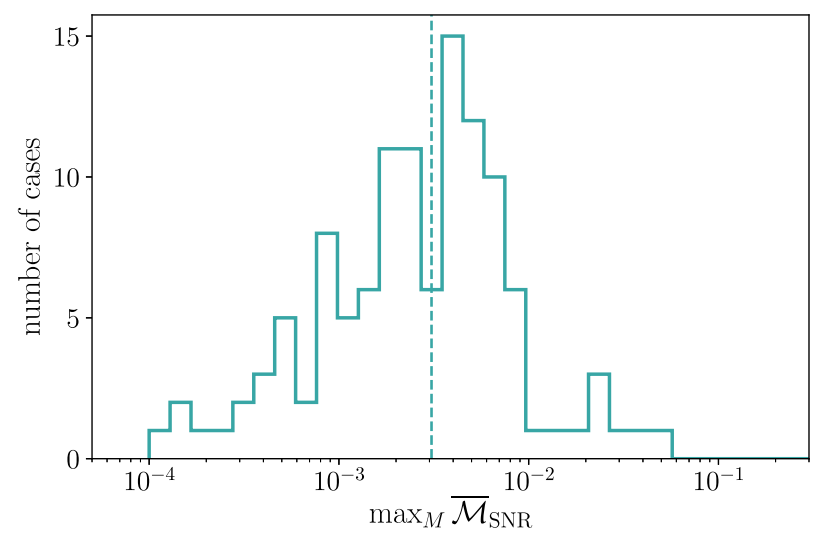

FIG. 2. The sky-and-polarization-averaged unfaithfulness between the highest and second highest resolutions in the NR simulation maximized over the total mass for the new $118 \mathrm{NR}$ precessing waveforms. The inclination used is $\pi / 3$. The vertical dashed line shows the median.

accuracy of SXS simulations in Ref. [52]. Therein, it was established that, when the nonastrophysical junk radiation perturbs the parameters of the simulation sufficiently, the different resolutions actually correspond to different physical systems. Thus, taking the difference between adjacent resolutions is no longer an appropriate estimate of the error.

We also find that the largest unfaithfulness occurs when the difference in parameters is largest, thus confirming that it is the difference in parameters that dominates the unfaithfulness.

\section{Effect of mode asymmetries in numerical-relativity waveforms}

The gravitational polarizations at time $t$ and location $\left(\varphi_{0}, l\right)$ on the coordinate sphere from the binary can be decomposed in -2-spin-weighted spherical harmonics, as follows

$$
\begin{aligned}
& h_{+}\left(\varphi_{0}, l ; t\right)-i h_{\times}\left(\varphi_{0}, l ; t\right) \\
& \quad=\sum_{\ell=2} \sum_{m=-\ell}^{m=+\ell}{ }_{-2} Y_{\ell m}\left(\varphi_{0}, l\right) h_{\ell m}(t) .
\end{aligned}
$$

For nonprecessing binaries, the invariance of the system under reflection across the orbital plane (taken to be the $x-y$ plane) implies $h_{\ell m}=(-1)^{\ell} h_{\ell-m}^{*}$. The latter is a very convenient relationship-for example it renders unnecessary to model modes with negative values of $m$. However, this relationship is no longer satisfied for precessing binaries.

As investigated in previous NR studies [25,82], we expect the asymmetries between opposite- $m$ modes to be small as compared to the dominant $(2,2)$-mode emission (at least during the inspiral) in a corotating frame that maximizes emission in the $(2, \pm 2)$ modes, also known as the maximum-radiation frame $[42,83]$. However, while the asymmetries are expected to be small during the inspiral, the difference in phase and amplitude between positive and negative $m$-modes might become non-negligible at merger.

As we discuss in the next section, when building multipolar waveforms (SEOBNRV4PHM) for precessing binaries by rotating modes from the coprecessing [21,23, 42-44] to the inertial frame of the observer, we shall neglect the mode asymmetries. To quantify the error introduced by this assumption, we proceed as follows. We first take NR waveforms in the coprecessing frame and construct symmetrized waveforms. Specifically, we consider the combination of waveforms in the coprecessing frame defined by (e.g., see Ref. [52])

$$
h_{\ell m}^{ \pm}=\frac{h_{\ell m}^{P} \pm h_{\ell-m}^{P *}}{2} \text {. }
$$

Note that if the assumption of conjugate symmetry holds (i.e., if $h_{\ell-m}^{P}=(-1)^{\ell} h_{\ell m}^{P *}$ ), then for even (odd) $\ell$ modes,
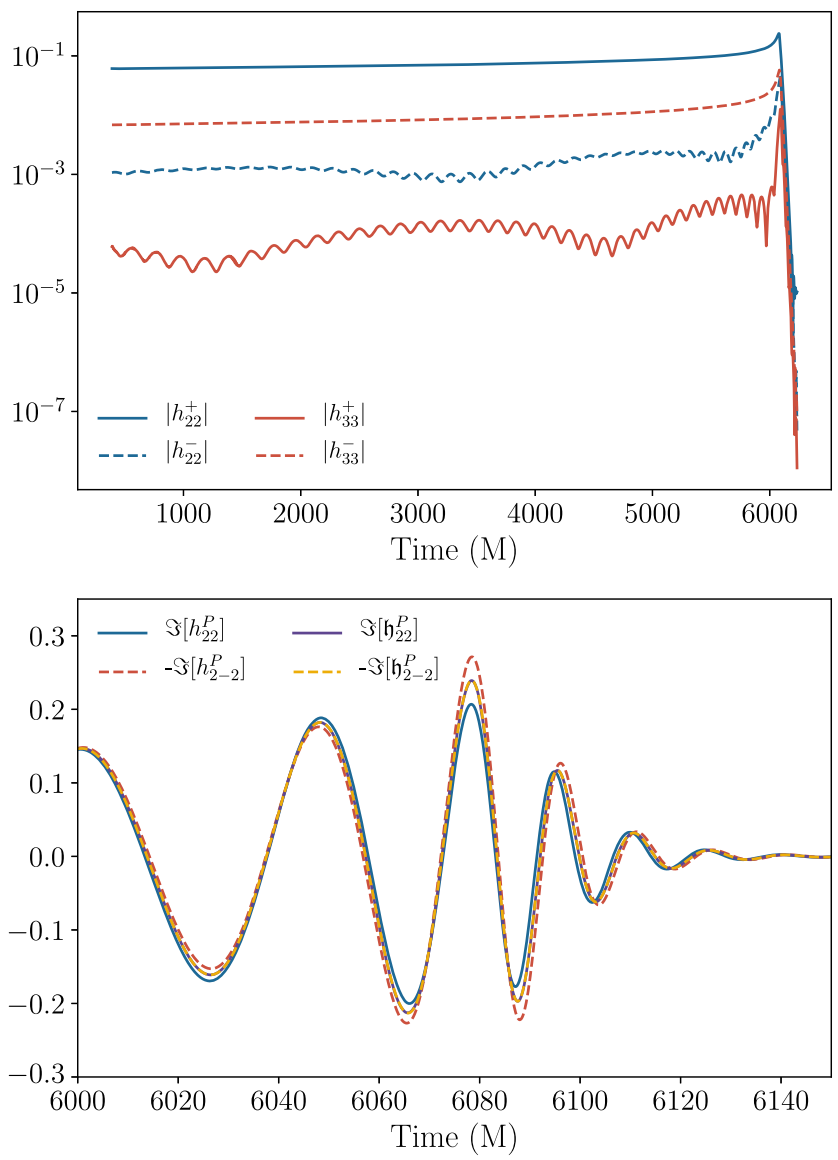

FIG. 3. Top: the behavior of $h_{\ell m}^{ \pm}$in the NR simulation PrecBBH000078. Note that especially during the inspiral, $\left|h_{22}^{+}\right|$ is much larger than $\left|h_{22}^{-}\right|$while $\left|h_{33}^{-}\right|$is much larger than $\left|h_{33}^{+}\right|$. Bottom: an example of waveform symmetrization for the same NR case, shown in the coprecessing frame. The symmetrized waveform obeys the usual conjugation symmetry as expected, and represents a reasonable average to the behavior of the unsymmetrized modes. 


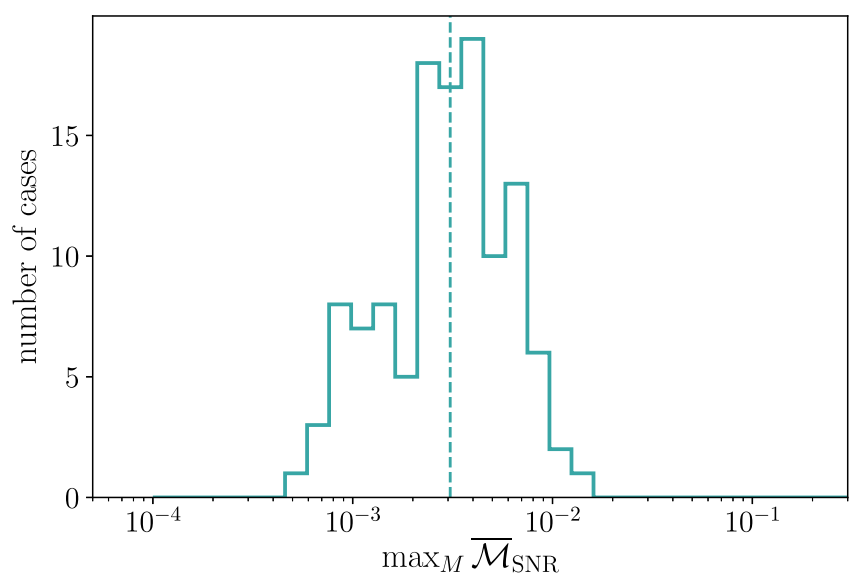

FIG. 4. The sky-and-polarization-averaged unfaithfulness between NR and symmetrized NR waveforms, maximized over the total mass for the new $118 \mathrm{NR}$ precessing waveforms. The inclination used is $\pi / 3$. The vertical dashed line shows the median.

$h_{\ell m}^{+}\left(h_{\ell m}^{-}\right)$is nonzero while the other component vanishes. If the assumption does not hold, it is still true that at given $\ell$, one of the components is much larger than the other, as shown in top panel of Fig. 3. Motivated by this, we define the symmetrized modes (for $m>0$ ) as [52]

$$
\mathfrak{h}_{\ell m}^{P}= \begin{cases}h_{\ell m}^{+} & \text {if } \ell \text { is even, } \\ h_{\ell m}^{-} & \text {if } \ell \text { is odd. }\end{cases}
$$

The other modes are constructed as $\mathfrak{h}_{\ell m}^{P}=(-1)^{\ell} \mathfrak{h}_{\ell-m}^{P *}$ for $m<0$, and we set $m=0$ modes to zero. The bottom panel of Fig. 3 shows an example of asymmetrized waveform for the case PrecBBH000078 of the SXS catalogue, in the coprecessing frame. It is obvious that the asymmetry between the modes has been removed and that the symmetrized waveform does indeed represent a reasonable "average" between the original modes. The symmetrized waveforms in the inertial frame are obtained by rotating the coprecessing frames modes back to the inertial frame.

In Fig. 4, we show the sky-and-polarization averaged unfaithfulness between the NR waveforms and the symmetrized waveforms described above, maximized over the total mass, including all modes available in the NR simulation, that is up to $\ell=8$. For the vast majority of the cases, the unfaithfulness is $\sim 0.5 \%$, and all cases have unfaithfulness below $2 \%$. This demonstrates that the effect of neglecting the asymmetry is likely subdominant to other sources of error such as the modeling of the waveform phasing, although the best way of quantifying the effect is to perform a Bayesian parameter-estimation study, which we leave to future work.

\section{MULTIPOLAR EOB WAVEFORMS FOR SPINNING, PRECESSING BINARY BLACK HOLES}

We briefly review the main ideas and building blocks of the EOB approach, and then describe an improved spinning, precessing EOBNR waveform model, which, for the first time, also contains multipole moments beyond the quadrupolar one. The model is already available in the LIGO Algorithm Library [84] under the name of SEOBNRV4 PHM We refer the reader to Refs. [26,33,46,85,86] for more details of the EOB framework and its most recent waveform models. Here we closely follow Ref. [33], highlighting when needed differences with respect to the previous precessing waveform model developed in Ref. [33] (SEOBNRv3 $\mathrm{P}^{4}$ ).

\section{A. Two-body dynamics}

The EOB formalism [87-90] can describe analytically the GW emission of the entire coalescence process, notably inspiral, merger and ringdown, and it can be made highly accurate by including information from NR. For the twobody conservative dynamics, the EOB approach relies on a Hamiltonian $H_{\mathrm{EOB}}$, which is constructed through: (i) the Hamiltonian $H_{\text {eff }}$ of a spinning particle of mass $\mu \equiv$ $m_{1} m_{2} /\left(m_{1}+m_{2}\right)$ and spin $\boldsymbol{S}_{*} \equiv \boldsymbol{S}_{*}\left(m_{1}, m_{2}, \boldsymbol{S}_{1}, \boldsymbol{S}_{2}\right)$ moving in an effective, deformed Kerr spacetime of mass $M \equiv$ $m_{1}+m_{2}$ and spin $\boldsymbol{S}_{\mathrm{Kerr}} \equiv \boldsymbol{S}_{1}+\boldsymbol{S}_{2}$ [91-93], and (ii) an energy map between $H_{\text {eff }}$ and $H_{\text {EOB }}$ [87]

$$
H_{\mathrm{EOB}} \equiv M \sqrt{1+2 \nu\left(\frac{H_{\mathrm{eff}}}{\mu}-1\right)}-M,
$$

where $\nu=\mu / M$ is the symmetric mass ratio. The deformation of the effective Kerr metric is fixed by requiring that at any given $\mathrm{PN}$ order, the $\mathrm{PN}$-expanded Hamiltonian $H_{\mathrm{EOB}}$ agrees with the PN Hamiltonian for BBHs [16]. In the EOB Hamiltonian used in this paper [92,93], the spin-orbit (spin-spin) couplings are included up to 3.5PN (2PN) order $[92,93]$, while the nonspinning dynamics is incorporated through $4 \mathrm{PN}$ order [46]. The dynamical variables in the EOB model are the relative separation $r$ and its canonically conjugate momentum $\boldsymbol{p}$, and the spins $\boldsymbol{S}_{1,2}$. The conservative EOB dynamics is completely general and can naturally accommodate precession $[26,33]$ and eccentricity [94-96].

When $\mathrm{BH}$ spins have generic orientations, both the orbital plane and the spins undergo precession about the total angular momentum of the binary, defined as $\boldsymbol{J} \equiv \boldsymbol{L}+\boldsymbol{S}_{1}+\boldsymbol{S}_{2}$, where $\boldsymbol{L} \equiv \boldsymbol{\mu} \boldsymbol{r} \times \boldsymbol{p}$. We also introduce the Newtonian orbital angular momentum $\boldsymbol{L}_{N} \equiv \mu \boldsymbol{r} \times \dot{\boldsymbol{r}}$, which at any instant of time is perpendicular to the binary's orbital plane. Black-hole spin precession is described by the following equations

\footnotetext{
${ }^{4}$ We note that whereas in LAL the name of this waveform approximant is SEOBNRV3, here we add a "P" to indicate "precession," making the notation uniform with respect to the most recent developed model SEOBNRV4P [33].
} 


$$
\frac{\mathrm{d} \boldsymbol{S}_{1,2}}{\mathrm{~d} t}=\frac{\partial H_{\mathrm{EOB}}}{\partial \boldsymbol{S}_{1,2}} \times \boldsymbol{S}_{1,2} .
$$

In the EOB approach, dissipative effects enter in the equations of motion through a nonconservative radiationreaction force that is expressed in terms of the GW energy flux through the waveform multipole moments [22,97-99] as

$$
\mathcal{F} \equiv \frac{\Omega}{16 \pi} \frac{\boldsymbol{p}}{|\boldsymbol{L}|} \sum_{\ell=2}^{8} \sum_{m=-\ell}^{\ell} m^{2}\left|d_{\mathrm{L}} h_{\ell m}\right|^{2},
$$

where $\Omega \equiv|\boldsymbol{r} \times \dot{\boldsymbol{r}}| /|\boldsymbol{r}|^{2}$ is the (angular) orbital frequency, $d_{\mathrm{L}}$ is the luminosity distance of the BBH to the observer, and the $h_{\ell m}$ 's are the GW multipole modes. As discussed in Refs. [45,46], the $h_{\ell m}$ used in the energy flux are not the same as those used for building the gravitational polarizations in the inertial frame, since the latter include the nonquasicircular corrections, which enforce that the SEOBNR waveforms at merger agree with the NR data, when available.

\section{B. Inspiral-plunge waveforms}

For the inspiral-plunge waveform, the EOB approach uses a factorized, resummed version [46,97-99] of the frequency-domain PN formulas of the modes [100,101]. As today, the factorized resummation has been developed only for quasicircular, nonprecessing BBHs [98,99], and it has been shown to improve the accuracy of the PN expressions in the test-particle limit, where one can compare EOB predictions to numerical solutions of the Regge-WheelerZerilli and Teukolsky equations $[32,49,102,103]$.

The radiation-reaction force $\mathcal{F}$ in Eq. (3.3) depends on the amplitude of the individual GW modes $\left|h_{\ell m}\right|$, which, in the nonprecessing case, are functions of the constant aligned-spin magnitudes $\chi_{1,2} \cdot \hat{\boldsymbol{L}}$. In the precessing case, these modes depend on time, as $\chi_{1,2}(t) \cdot \hat{\boldsymbol{L}}(t)$, and they depend on the generic, precessing orbital dynamics through the radial separation $r$ and orbital frequency $\Omega$, which carry modulations due to spin-spin couplings whenever precession is present. However, we stress that with this choice of the radiation-reaction force and waveform model, not all spin-precession effects are included, since the PN formulas of the modes [100] also contain terms that depend on the in-plane spin components.

For data-analysis purposes, we need to compute the GW polarizations in the inertial-frame of the observer (or simply observer's frame). We denote quantities in this frame with the superscript $I$. The observer's frame is defined by the $\operatorname{triad}\left\{\hat{\boldsymbol{e}}_{(i)}^{I}\right\}(i=1,2,3)$, where $\hat{\boldsymbol{e}}_{(1)}^{I} \equiv \hat{\boldsymbol{r}}(0), \hat{\boldsymbol{e}}_{(3)}^{I} \equiv \hat{\boldsymbol{L}}_{N}(0)$ and $\hat{\boldsymbol{e}}_{(2)}^{I} \equiv \hat{\boldsymbol{e}}_{(3)}^{I} \times \hat{\boldsymbol{e}}_{(1)}^{I}$. Moreover, in this frame, the line of sight of the observer is parametrized as $\hat{N} \equiv$ $\left(\sin \imath \cos \phi_{o}, \sin \imath \sin \phi_{o}, \cos \imath\right.$ ) (see Fig. 5). We also introduce the observer's frame with the polarization basis $\left\{\hat{\boldsymbol{e}}_{(1)}^{r}, \hat{\boldsymbol{e}}_{(2)}^{r}\right\} \quad$ such that $\hat{\boldsymbol{e}}_{(1)}^{r} \equiv\left(\hat{\boldsymbol{e}}_{(3)}^{I} \times \hat{\boldsymbol{N}}\right) /\left|\hat{\boldsymbol{e}}_{(3)}^{I} \times \hat{\boldsymbol{N}}\right|$ and $\hat{\boldsymbol{e}}_{(2)}^{r} \equiv \hat{\boldsymbol{N}} \times \hat{\boldsymbol{e}}_{(1)}^{r}$, which spans the plane orthogonal to $\hat{\boldsymbol{N}}$.

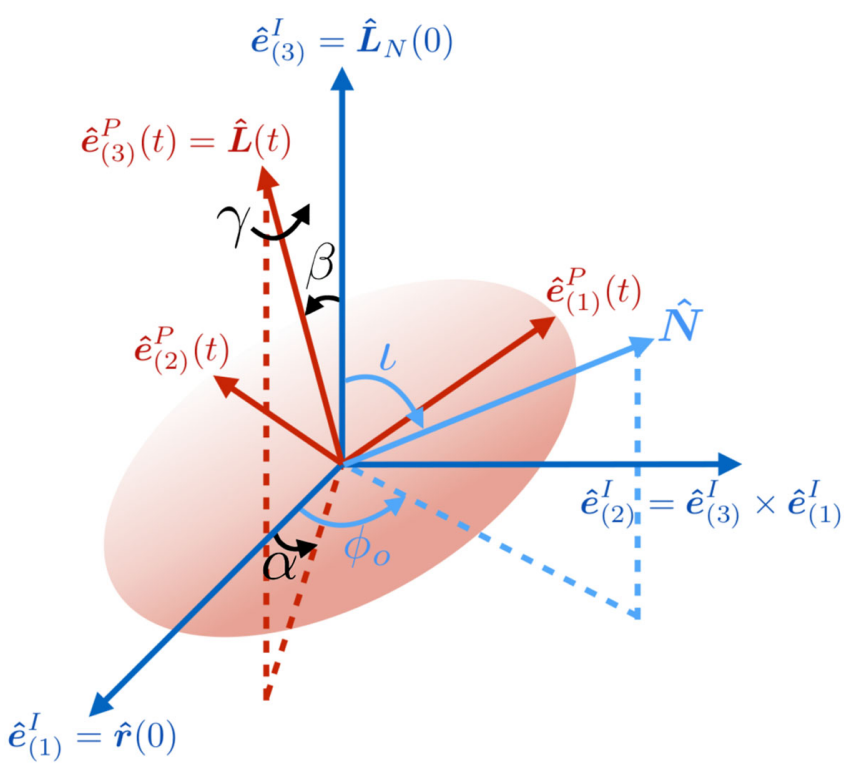

FIG. 5. Frames used in the construction of the SEOBNRv4 PHM model: the observer's frame (blue), defined by the directions of the initial orbital angular momentum $\hat{\boldsymbol{L}}(0)$ and separation $\boldsymbol{r}(0)$, and coprecessing frame (red), instantaneously aligned with $\hat{\boldsymbol{L}}(t)$ and described by the Euler angles $(\alpha, \beta, \gamma)$ (see text below for details).

To compute the observer's-frame modes $h_{\ell m}^{I}$ during the inspiral-plunge stage, it is convenient to introduce a noninertial reference frame that tracks the motion of the orbital plane, the so-called coprecessing frame (superscript $P$ ), described by the triad $\left\{\hat{\boldsymbol{e}}_{(i)}^{P}\right\}(i=1,2,3)$. At each instant, its $z$-axis is aligned with $\hat{\boldsymbol{L}}: \hat{\boldsymbol{e}}_{(3)}^{P} \equiv \hat{\boldsymbol{L}}(t) .{ }^{5}$ In this frame, the BBH is viewed face-on at all times, and the GW radiation looks very much nonprecessing $[21,23,42-44]$. The other two axes lie in the orbital plane and are defined such as they minimize precessional effects in the precessingframe modes $h_{\ell m}^{P}$ [21,42]. After introducing the vector $\boldsymbol{\Omega}_{e} \equiv \hat{\boldsymbol{L}} \times \mathrm{d} \hat{\boldsymbol{L}} / \mathrm{d} t$, we enforce the minimum-rotation condition by requiring that $\mathrm{d} \hat{\boldsymbol{e}}_{(1),(2)}^{P} / \mathrm{d} t=\boldsymbol{\Omega}_{e} \times \hat{\boldsymbol{e}}_{(1),(2)}^{P}$ and $\hat{\boldsymbol{e}}_{(1),(2)}^{P}(0)=\hat{\boldsymbol{e}}_{(1),(2)}^{I}$ (see also Fig. 5). As usual, we parametrize the rotation from the precessing to the observer's frame through time-dependent Euler angles $(\alpha(t), \beta(t), \gamma(t))$, which we compute using Eqs. (A4)-(A6) in Appendix A of Ref. [33]. We notice that the minimum-rotation condition can also be expressed through the following differential equation for $\gamma: \dot{\gamma}=-\dot{\alpha} \cos \beta$ with $\gamma(0)=-\alpha(0)=\pi / 2$.

We compute the precessing-frame inspiral-plunge modes just like we do for the GW flux, namely by evaluating the factorized, resummed nonprecessing multipolar waveforms along the EOB precessing dynamics, and employing the time-dependent spin projections $\chi_{1,2}(t) \cdot \hat{\boldsymbol{L}}(t)$. Finally, the observer's-frame inspiral-plunge modes are obtained by

\footnotetext{
${ }^{5}$ Note that in Ref. [33], the $z$-axis is aligned with $\hat{\boldsymbol{L}}_{\mathrm{N}}$ instead of $\hat{\boldsymbol{L}}$.
} 
rotating the precessing-frame inspiral-plunge modes using Eq. (A13) in Appendix A of Ref. [33].

Following Ref. [46], where an EOBNR nonprecessing multipolar waveform model was developed (SEOBNRV4HM), here we include in the precessing frame of the SEOBNRV4PHM model the $(2, \pm 2),(2, \pm 1),(3, \pm 3)$, $(4, \pm 4)$ and $(5, \pm 5)$ modes, and make the assumption $h_{l-m}^{P}=(-1)^{l} h_{l m}^{P *}$. As shown in Sec. II D, we expect that inaccuracies due to neglecting mode asymmetries should remain mild, or at most at the level of other modeling errors.

\section{Merger-ringdown waveforms}

The description of a BBH as a system composed of two individual objects is of course valid only up to the merger. After that point, the EOB model builds the GW emission (ringdown stage) via a phenomenological model of the quasinormal modes (QNMs) of the remnant $\mathrm{BH}$, which forms after the coalescence of the progenitors. The QNM frequencies and decay times are known (tabulated) functions of the mass $M_{f}$ and spin $S_{f} \equiv M_{f}^{2} \chi_{f}$ of the remnant $\mathrm{BH}$ [104]. Since the QNMs are defined with respect to the direction of the final spin, the specific form of the ringdown signal, as a linear combination of QNMs, is formally valid only in an inertial frame whose $z$-axis is parallel to $\chi_{f}$.

A novel feature of the SEOBNRV4 PHM waveform model presented here is that we attach the merger-ringdown waveform (notably each multipole mode $h_{\ell m}^{\text {mergr-RD }}$ ) directly in the coprecessing frame, instead of the observer's frame. As a consequence, we can employ here the mergerringdown multipolar model developed for nonprecessing BBHs (SEOBNRV4HM) in Ref. [46] (see Sec. IV E therein for details). By contrast, in the SEOBNRV3P waveform model [33], the merger-ringdown waveform was built as a superposition of QNMs in an inertial frame aligned with the direction of the remnant spin. This construction was both more complicated to implement and more prone to numerical instabilities.

To compute the waveform in the observer's frame, our approach requires a description of the coprecessing frame Euler angles $(\alpha, \beta, \gamma)$ that extends beyond the merger. To prescribe this, we take advantage of insights from NR simulations [24]. In particular, it was shown that the coprecessing frame continues to precess roughly around the direction of the final spin with a precession frequency approximately equal to the differences between the lowest overtone of the $(2,2)$ and $(2,1)$ QNM frequencies, while the opening angle of the precession cone decreases somewhat at merger. We find that this behavior is qualitatively correct for the NR waveforms used for comparison in this paper.

To keep our model generic for a wide range of mass ratios and spins, we need an extension of the behavior noticed in Ref. [24] to the retrograde case, where the remnant spin is negatively aligned with the orbital angular momentum at merger. Such configurations can occur for high mass-ratio binaries, when the total angular momentum
$\boldsymbol{J}$ is dominated by the spin of the primary $\boldsymbol{S}_{1}$ instead of the orbital angular momentum $\boldsymbol{L}$. This regime is not well explored by NR simulations, and includes in particular systems presenting transitional precession [15]. In our model we keep imposing simple precession around the direction of the remnant spin at a rate $\omega_{\text {prec }} \geq 0$, but we distinguish two cases depending on the direction of the final spin $\chi_{f}$ (approximated by the total angular momentum $\boldsymbol{J}=\boldsymbol{L}+\boldsymbol{S}_{1}+\boldsymbol{S}_{2}$ at merger) relative to the final orbital angular momentum $\boldsymbol{L}_{f}$ :

$\dot{\alpha}=\omega_{\text {prec }}= \begin{cases}\omega_{22}^{\mathrm{QNM}}\left(\chi_{f}\right)-\omega_{21}^{\mathrm{QNM}}\left(\chi_{f}\right) & \text { if } \chi_{f} \cdot \boldsymbol{L}_{f}>0 \\ \omega_{2-1}^{\mathrm{QNM}}\left(\chi_{f}\right)-\omega_{2-2}^{\mathrm{QNM}}\left(\chi_{f}\right) & \text { if } \chi_{f} \cdot \boldsymbol{L}_{f}<0\end{cases}$

where $\chi_{f}=\left|\chi_{f}\right|$, and the zero-overtone QNM frequencies for negative $m$ are taken on the branch $\omega_{l m}^{\mathrm{QNM}}>0$ that continuously extends the $m>0, \omega_{l m}^{\mathrm{QNM}}>0$ branch [104] (the QNM refers to zero overtone). In both cases, $\dot{\alpha} \geq 0$. We do not attempt to model the closing of the opening angle of the precession cone and simply consider it to be constant during the post-merger phase, $\beta=$ const. The third Euler angle $\gamma$ is then constructed from the minimal rotation condition $\dot{\gamma}=-\dot{\alpha} \cos \beta$. The integration constants are determined by matching with the inspiral at merger. We find that the behavior of Eq. (3.4) in the case $\chi_{f} \cdot \boldsymbol{L}_{f}<0$ is qualitatively consistent with an NR simulation investigated by one of us [105]. However, we stress that this prescription for the retrograde case is much less tested than for the prograde case.

Furthermore, one crucial aspect of the above construction is the mapping from the binary's component masses and spins to the final mass and spin, which is needed to compute the QNM frequencies of the merger remnant. Many groups have developed fitting formulae based on a large number of NR simulations (e.g., see Ref. [106] for an overview). To improve the agreement of our EOB merger-ringdown model with NR, and to ensure agreement in the aligned-spin limit with SEOBNRV4 [45] and SEOBNRV4HM [46], we employ the fits from Hofmann et al. [107]. In Fig. 6 we compare the performance of the fit used in the previous EOB precessing model SEOBNRv3P $[26,33,86]$ to the fit from Hofmann et al. that we adopt for SEOBNRV4 PHM. It is clear that the new fit reproduces NR data much better. This in turn improves the correspondence between NR and EOB QNM frequencies.

For the final mass we employ the same fit as in previous EOB models, and we provide it here since it was not given explicitly anywhere before:

$$
\begin{aligned}
\frac{M_{f}}{M}= & 1-\left\{\left[1-E_{\mathrm{ISCO}}(a)\right] \nu+16 \nu^{2}[0.00258\right. \\
& -\frac{0.0773}{\left[a(1+1 / q)^{2} /\left(1+1 / q^{2}\right)-1.6939\right]} \\
& \left.\left.-\frac{1}{4}\left(1-E_{\mathrm{ISCO}}(a)\right)\right]\right\},
\end{aligned}
$$




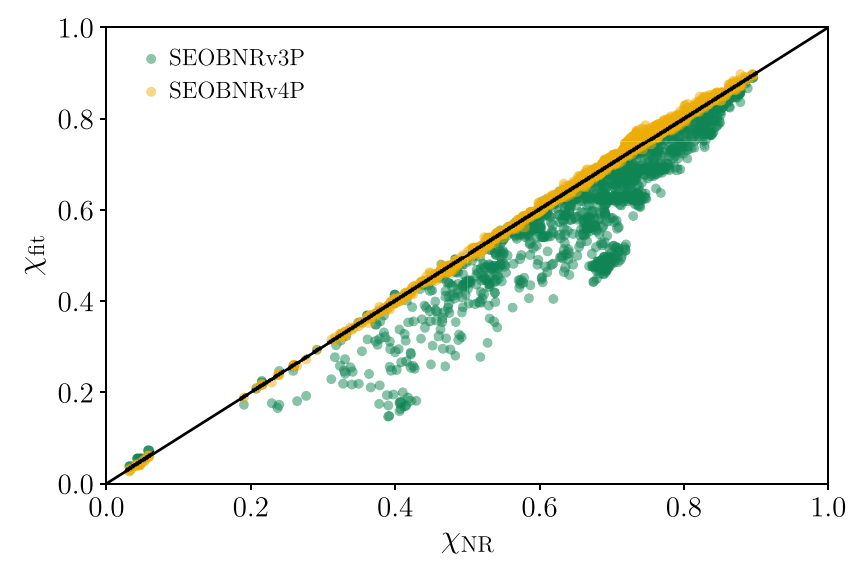

FIG. 6. Comparison of the magnitude of the final spin between the SEOBNRV3P and SEOBNRV4P models and NR results. For simplicity, the fits are evaluated using the NR data at the relaxed time. The black line is the identity. It is obvious that SEOBNRV4 P gives final-spin magnitudes much closer to the NR values.

where $a=\hat{\boldsymbol{L}} \cdot\left(\chi_{1}+\chi_{2} / q^{2}\right) /(1+1 / q)^{2}$, and $E_{\mathrm{ISCO}}(a)$ is the binding energy of the Kerr spacetime at the innermost stable circular orbit [108].

Finally, for precessing binaries, the individual components of the spins vary with time. Therefore, in applying the fitting formulae to obtain final mass and spin, one must make a crucial choice in selecting the time during the inspiral stage at which the spin directions are evaluated. In fact, even if one considers a given physical configuration, evaluating the final spin formulae with spin directions from different times yields different final spins and consequently different waveforms. We choose to evaluate the spins at a time corresponding to the separation of $r=10 \mathrm{M}$. This choice is guided by two considerations: by the empirical finding of good agreement with NR (e.g., performing better than using the time at which the inspiral-plunge waveform is attached to the mergerringdown waveform [46]), and by the restriction that the waveform must start at $r>10.5 \mathrm{M}$ in order to have small initial eccentricity [33]. Thus, our choice ensures that a given physical configuration always produces the same waveform regardless of the initial starting frequency.

To obtain the inspiral-merger-ringdown modes in the inertial frame, $h_{\ell m}^{\mathrm{I}}$, we rotate the inspiral-merger-ringdown modes $h_{\ell m}^{\mathrm{P}}$ from the coprecessing frame to the observer's frame using the rotation formulas and Euler angles in Appendix A of Ref. [33]. The inertial frame polarizations then read

$$
h_{+}^{\mathrm{I}}\left(\varphi_{0}, l ; t\right)-i h_{\times}^{\mathrm{I}}\left(\varphi_{0}, l ; t\right)=\sum_{\ell, m}{ }_{-2} Y_{\ell m}\left(\varphi_{0}, \imath\right) h_{\ell m}^{\mathrm{I}}(t) .
$$

\section{On the fits of calibration parameters in presence of precession}

The SEOBNRV4 PHM waveform model inherits the EOB Hamiltonian and GW energy flux from the aligned-spin model SEOBNRv4 [45], which features higher (yet unknown) $\mathrm{PN}$-order terms in the dynamics calibrated to NR waveforms. These calibration parameters were denoted $K, d_{\mathrm{SO}}$ and $d_{\mathrm{SS}}$ in Ref. [45], and were fitted to NR and Teukolsky-equation-based waveforms as polynomials in $\nu$, $\chi$ where $\chi \equiv S_{\text {Kerr }}^{z} /(1-2 \nu)$ with $S_{\text {Kerr }}=\mathbf{S}_{1}+\mathbf{S}_{2}$ the spin of the EOB background spacetime. In contrast to the SEOBNRV3P waveform model, which used the EOB Hamiltonian and GW energy flux from the aligned-spin model SEOBNRv2 [86], the fits in Ref. [45] include odd powers of $\chi$ and thus the sign of $\chi$ matters when the BHs precess.

The most natural way to generalize these fits to the precessing case is to project $\boldsymbol{S}_{\text {Kerr }}$ onto the orbital angular momentum $\hat{\boldsymbol{L}}$ in the usual spirit of reducing precessing quantities to corresponding aligned-spin ones. To test the impact of this prescription, we compute the sky-andpolarization-averaged unfaithfulness with the set of 118 NR simulations described in Sec. II, and find that while the majority of the cases have low unfaithfulness $(\sim 1 \%)$, there are a handful of cases where it is significant $(\sim 10 \%)$, with many of them having large in-plane spins.

To eliminate the high mismatches, we introduce the augmented spin that includes contribution of the in-plane spins:

$$
\tilde{\chi}=\frac{\boldsymbol{S}_{\mathrm{Kerr}} \cdot \boldsymbol{L}}{1-2 \nu}+\alpha \frac{\left(\boldsymbol{S}_{1}^{\perp}+\boldsymbol{S}_{2}^{\perp}\right) \cdot \boldsymbol{S}_{\mathrm{Kerr}}}{\left|\boldsymbol{S}_{\mathrm{Kerr}}\right|(1-2 \nu)} .
$$

Here $\boldsymbol{S}_{i}^{\perp} \equiv \boldsymbol{S}_{i}-\left(\boldsymbol{S}_{i} \cdot \boldsymbol{L}\right) \boldsymbol{L}$ and $\alpha$ is a positive coefficient to be determined. Note that the extra term in the definition of the augmented spin $\geq 0$ for any combination of the spins. We set $\tilde{\chi}=0$ when $S_{\text {Kerr }}=0$. Fixing $\alpha=1 / 2$ insures that the augmented spin obeys the Kerr bound. Using the augmented spin eliminates all mismatches above $6 \%$, and thus greatly improves the agreement of the model with NR data.

\section{COMPARISON OF MULTIPOLAR PRECESSING MODELS TO NUMERICAL- RELATIVITY WAVEFORMS}

To assess the impact of the improvements incorporated in the SEOBNRV4 PHM waveform model, we compare this model and other models publicly available in LAL (see Table I) to the set of simulations described in Sec. II, as well as to all publicly available precessing SPEC simulations. ${ }^{6}$

We start by comparing in Fig. 7, the precessing NR waveform PrecBBH0 0078 with mass ratio 4, BH's spin magnitudes 0.7 , total mass $M=70 M_{\odot}$ and modes $\ell \leq 4$ from the new 118 SXS catalog (see Appendix B) to the precessing waveforms IMRPhenomPV3 and SEOBNRV4P with modes $\ell=2$ (upper panels), and to the precessing multipolar waveforms IMRPhenomPv3HM and

\footnotetext{
${ }^{6}$ The list of all SXS simulations used can be found in https:// arxiv.org/src/1904.04831v2/anc/sxs_catalog.json
} 


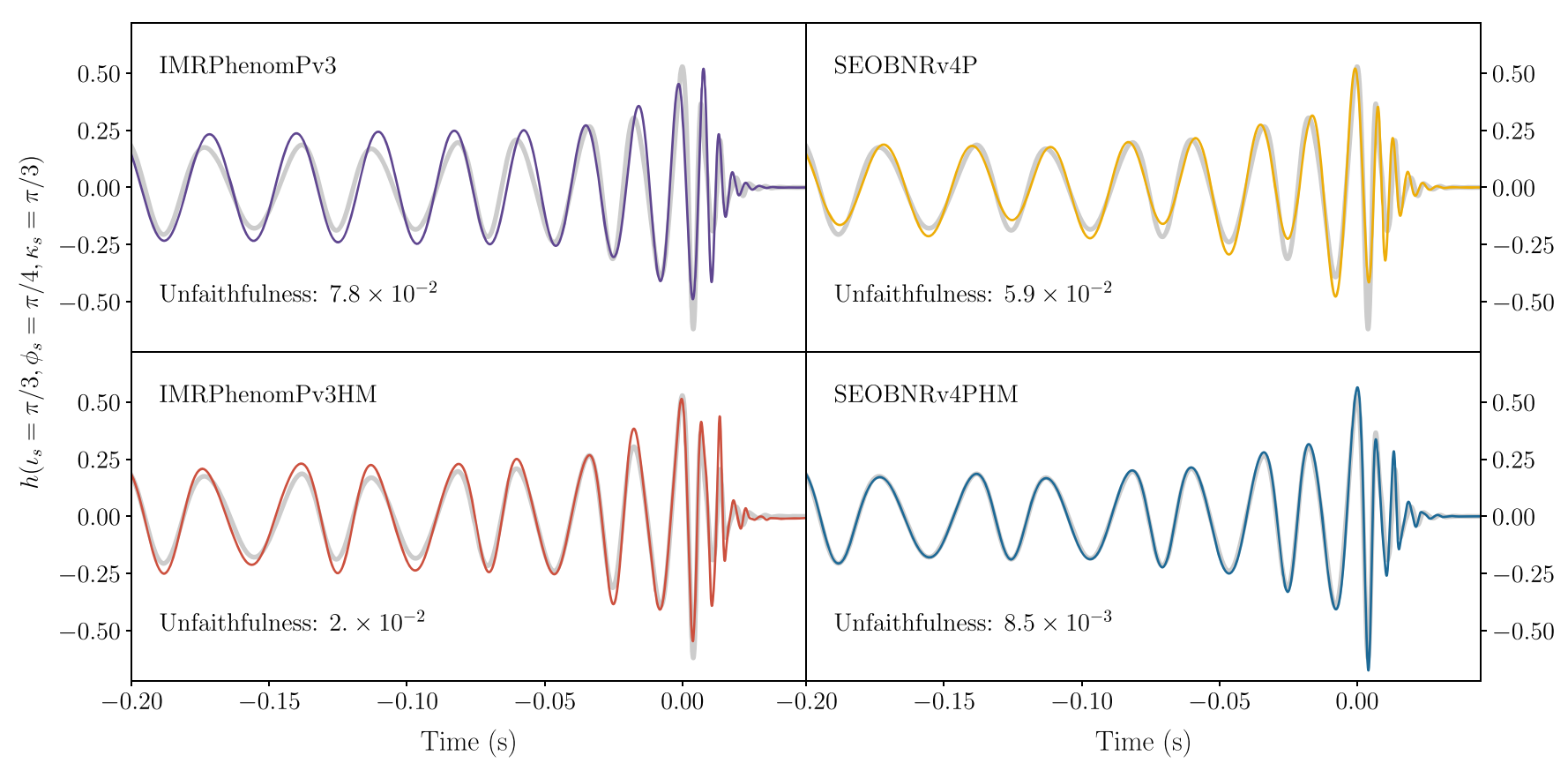

FIG. 7. Time-domain comparison of state-of-the art waveform models to the NR waveform PrecBBH0 0078 with mass ratio 4, BH's spins 0.7 and total mass $M=70 M_{\odot}$. The source parameters are $\imath_{s}=\pi / 3, \phi_{s}=\pi / 4, \kappa_{s}=\pi / 4$. The NR waveform includes all modes up to and including $\ell=4$, and extends for $44 \mathrm{GW}$ cycles before merger. For models that include only $\ell=2$ modes, the unfaithfulness are several percent $8 \%$ for IMRPhenomPv3 and $6 \%$ for SEOBNRV4P. Meanwhile, adding the higher mode content drastically improves the agreement, with mismatches going down to $2 \%$ for IMRPhenomPV3HM and $1 \%$ for SEOBNRV4 PHM. The agreement is particular good for SEOBNRV4PHM, which reproduces the higher mode features at merger and ringdown faithfully.

SEOBNRV4 PHM (lower panels). This NR waveform is the most "extreme" configuration from the new set of waveforms and has about $44 \mathrm{GW}$ cycles before merger, and the plot only shows the last 7 cycles. More specifically, we plot the detector response function given in Eq. (2.5), but we leave out the overall constant amplitude. We indicate on the panels the unfaithfulness for the different cases. We note the improvement when including modes beyond the quadrupole. SEOBNRV4 PHM agrees particularly well to this NR waveform, reproducing accurately the higher-mode features throughout merger and ringdown.

We now turn to the public precessing SXS NR catalog of 1404 waveforms. First, to quantify the performance of the new precessing waveform model SEOBNRV $4 \mathrm{P}$ with respect to previous precessing models used in LIGO and Virgo inference studies, we compute the unfaithfulness ${ }^{7}$ against the precessing NR catalog, including only the dominant $\ell=2$ multipoles in the coprecessing frame. Figure 8 shows the histograms of the largest mismatches when the binary total mass varies in the range $[20,200] M_{\odot}$. Here, we also consider the precessing waveform models used in the first GW Transient Catalog [5] of the LIGO and Virgo collaboration (i.e., SEOBNRV3P and IMRPhenomPv2). Two trends are apparent: first, SEOBNRv3P and

\footnotetext{
${ }^{7}$ We always use the sky-and-polarization averaged, SNRweighted faithfulness or unfaithfulness $\overline{\mathcal{M}}_{\text {SNR }}$ unless otherwise stated.
}

IMRPhenomPv2 distributions are broadly consistent, with both models having mismatches which extend beyond $10 \%$, although SEOBNRV3 has more cases at lower unfaithfulness; second, SEOBNRV4P has a distribution which is shifted to much lower values of the unfaithfulness and does not include outliers with the largest unfaithfulness below $7 \%$.

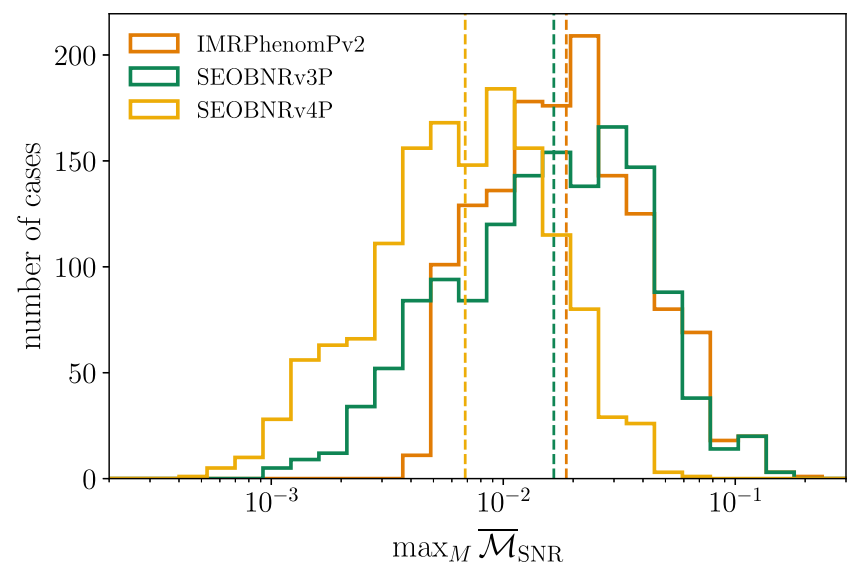

FIG. 8. Sky-and-polarization averaged, SNR weighted unfaithfulness for an inclination $l=\pi / 3$ between NR waveforms with $\ell=2$ and SEOBNRv4P, and also SEOBNRV3P and IMRPhenomPv2, which were used in LIGO/Virgo publications. The vertical dashed lines show the medians. It is evident the better performance of the newly developed precessing model SEOBNRV4P. 


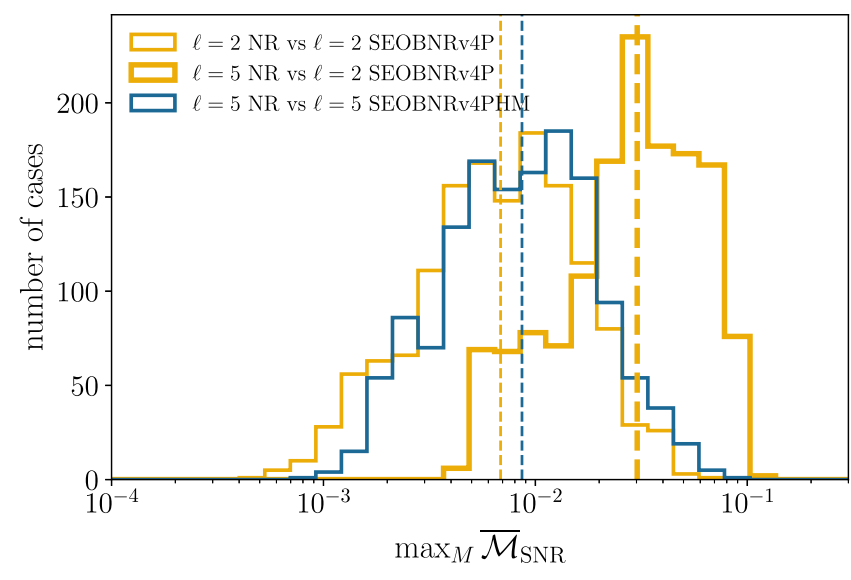

FIG. 9. Sky-and-polarization averaged, SNR weighted unfaithfulness for an inclination $l=\pi / 3$ between NR waveforms and SEOBNRV4PHM, including and omitting higher modes. The vertical dashed lines show the medians. Not including higher modes in the model results in high unfaithfulness. However, when they are included, the unfaithfulness between SEOBNRV4 PHM and $\mathrm{NR}$ is essentially at the same level as when only $\ell=2$ modes are compared (see Fig. 8).

Next, we examine the importance of higher modes. To do so, we use SEOBNRV4PHM with and without the higher modes, while always including all modes up to $\ell=5$ in the NR waveforms. As can be seen in Fig. 9, if higher modes are omitted, the unfaithfulness can be very large, with a significant number of cases having unfaithfulness $>7 \%$, as has been seen in many past studies. On the other hand, once higher modes are included in the model, the distribution of mismatches becomes much narrower, with all mismatches below 9\%. Furthermore, the distribution now closely resembles the distribution of mismatches when only $\ell=2$ modes were included in the NR waveforms. Thus, we see that higher modes play an important role and are accurately captured by SEOBNRV4 PHM waveform model.

Moreover, in Fig. 10 we display, for a specific choice of the inclination, the unfaithfulness versus the binary's total mass between the public precessing SXS NR catalog and SEOBNRV4PHM and IMRPhenomPv3HM. We highlight with curves in color the NR configurations having worst maximum mismatches for the two classes of approximants. For the majority of cases, both models have unfaithfulness below 5\%, but SEOBNRV4 PHM has no outliers beyond $10 \%$ and many more cases at lower unfaithfulness $\left(<2 \times 10^{-3}\right)$. We find that the large values of unfaithfulness above $10 \%$ for IMRPhenomPv3HM come from simulations with $q \gtrsim 4$ and large antialigned primary spin, i.e., $\chi_{1}^{z}=-0.8$. An examination of the waveforms in this region reveals that unphysical features develop in the waveforms, with unusual oscillations both in amplitude and phase. For lower spin magnitudes these features are milder, and disappear for spin magnitudes $\lesssim 0.65$. These features are present also in IMRPhenomPv 3 and are thus connected to the precession dynamics, a region already known to potentially pose a challenge when modeling the precession dynamics as suggested in Ref. [109], and adopted in Ref. [39].

We now focus on the comparisons with the 118 SXS NR waveforms produced in this paper. In Fig. 11 we show the unfaithfulness for IMRPhenomPV3 (HM) and SEOBNRV4P (HM) in the left (right) panels. We compare waveforms without higher modes, to NR data that has only the $\ell=2$ modes, and the other models to NR data with $\ell \leq 4$ modes. The performance of both waveform models on this new NR data set is largely comparable to what was found for the public catalog. Both families perform well on average, with most cases having unfaithfulness below $2 \%$ for models without higher modes and 3\% for models with higher modes. However, for some configurations IMRPhenomPv3 (HM) reaches unfaithfulness values above $3 \%$ for total masses below $125 M_{\odot}$. Once again, the overall distribution is shifted to lower unfaithfulness values for SEOBNRv4P (HM) .

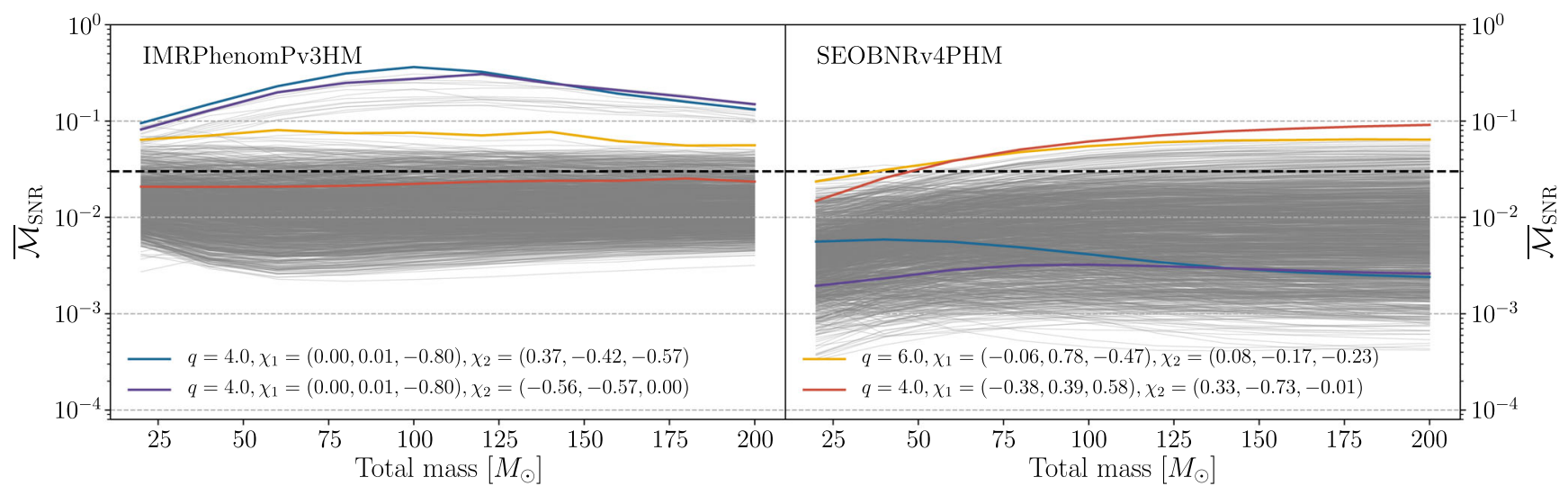

FIG. 10. The sky-and-polarization averaged, SNR-weighted unfaithfulness as a function of binary's total mass for inclination $l=\pi / 3$, between IMRPhenomPv3HM and NR (left) and SEOBNRV4 PHM and NR (right) for 1404 quasi-circular precessing BBH simulations in the SXS public catalog. The colored lines highlight the cases with the worst maximum mismatches for both models. Note that for the majority of cases, both models have unfaithfulness below 5\%, but SEOBNRV4 PHM has no outliers beyond 10\% and many more cases at lower unfaithfulness. 


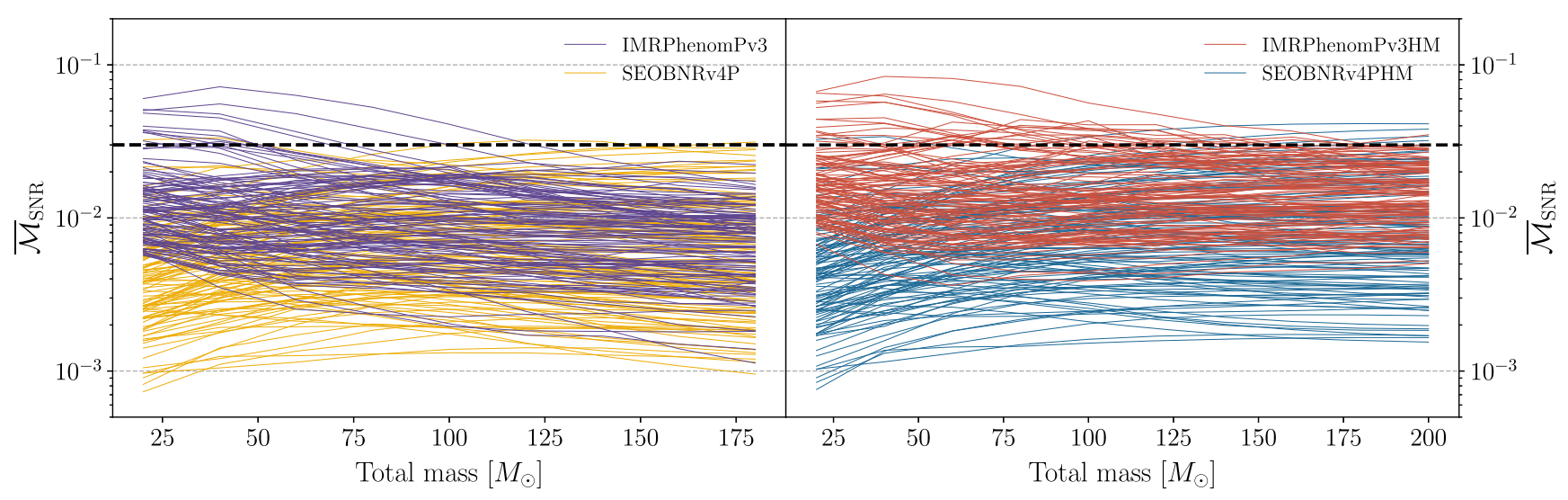

FIG. 11. The sky-and-polarization averaged, SNR-weighted unfaithfulness as a function of binary's total mass for inclination $l=\pi / 3$, between IMRPhenomPV3 and SEOBNRV4P and NR (left), and IMRPhenomPV3HM and SEOBNRV4PHM and NR (right) for the 118 SXS NR waveforms described in Appendix B. The NR data has $\ell=2$ modes for the left panel, while all modes up to and including $\ell=4$ in the right panel. The unfaithfulness is low using both waveform families, however, SEOBNRv4P (HM) has fewer cases above $3 \%$, and the distribution is consistently shifted to lower values of unfaithfulness.

When studying the distribution of unfaithfulness for these 118 cases across parameter space, it is useful to introduce the widely used effective $\chi_{\text {eff }}[90,110,111]$ and precessing $\chi_{p}$ [112] spins. These capture the leading order aligned-spin and precession effects respectively, and are defined as

$$
\begin{aligned}
& \chi_{\text {eff }}=\frac{\left(m_{1} \chi_{1}+m_{2} \chi_{2}\right)}{m_{1}+m_{2}} \cdot \hat{\boldsymbol{L}}_{N}, \\
& \chi_{p}=\frac{1}{B_{1} m_{1}^{2}} \max \left(B_{1} m_{1}^{2} \chi_{1 \perp}, B_{2} m_{2}^{2} \chi_{2 \perp}\right),
\end{aligned}
$$

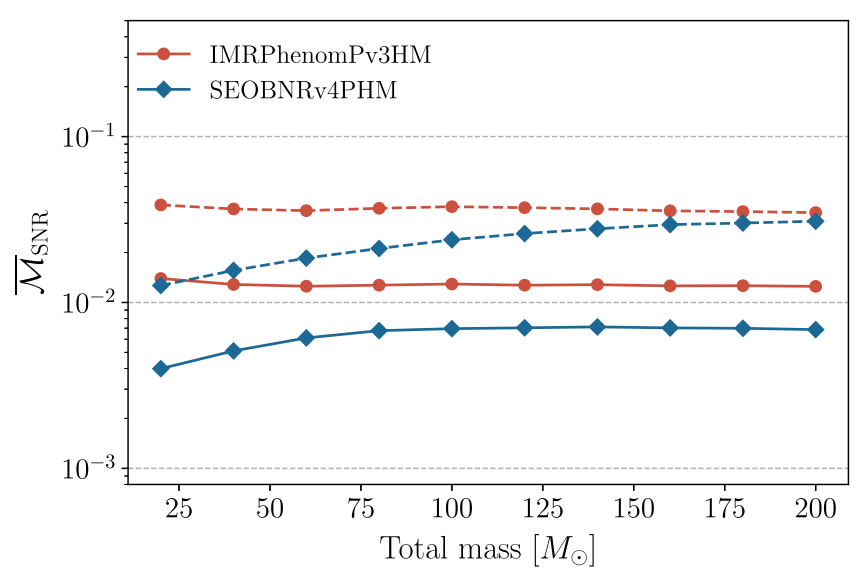

FIG. 12. Summary of unfaithfulness as a function of the total mass, for all NR simulations considered as shown in Fig. 10 and Fig 11. The solid (dotted) line represents the median $(95 \%$ percentile) of all cases. For all total masses, we find that the median mismatch with SEOBNRV4 PHM is lower than $1 \%$, about a factor of 2 lower than IMRPhenomPv3HM. The 95th-percentile shows a stronger dependence on total mass for SEOBNRV4 PHM, with mismatches lower than IMRPhenomPv3HM at low and medium total masses, becoming comparable at the highest total masses. where with $B_{1}=2+3 m_{2} / m_{1}, B_{2}=2+3 m_{1} / m_{2}$ and we indicate with $\chi_{i \perp}$ the projection of the spins on the orbital plane. We find that the unfaithfulness shows 2 general trends. First, it tends to increase with increasing $\chi_{\text {eff }}$ and $\chi_{p}$. Second, that cases with positive $\chi_{\text {eff }}$ (i.e., aligned with Newtonian orbital angular momentum) tend to have larger unfaithfulness. This is likely driven by the fact that inspiral is longer for such cases and the binary merges at higher frequency. We do not find any other significant trends based on spin directions. It is interesting to note that the distribution of mismatches from the 118 cases is quite similar to the distribution from the much larger public catalog. This suggests that the 118 cases do indeed explore many different regimes of precession.

To further quantify the results of the comparison between the precessing multipolar models SEOBNRV4PHM and IMRPhenomPv3HM and the NR waveforms, we show in Figs. 12 and 13 the median and 95\%-percentile of all cases, and the highest unfaithfulness as function of the total mass, respectively. These studies also demonstrate the better performance of SEOBNRV4PHM with respect to IMRPhenomPv3HM.

To summarize the performance against the entire SXS catalog (including the new 118 precessing waveforms) we find that for SEOBNRV4PHM, out of a total of $1523 \mathrm{NR}$ simulations we have considered, 864 cases (57\%) have a maximum unfaithfulness less than $1 \%$, and 1435 cases (94\%) have unfaithfulness less than 3\%. Meanwhile for IMRPhenomPv3 HM the numbers become 300 cases (20\%) below $1 \%, 1256$ cases $(83 \%)$ below $3 \%{ }^{8}$ The accuracy of the semianalytical waveform models can be improved in

\footnotetext{
${ }^{8}$ Due to technical details of the IMRPhenomPv3HM model, the total number of cases analyzed for this model is 1507 instead of 1523.
} 


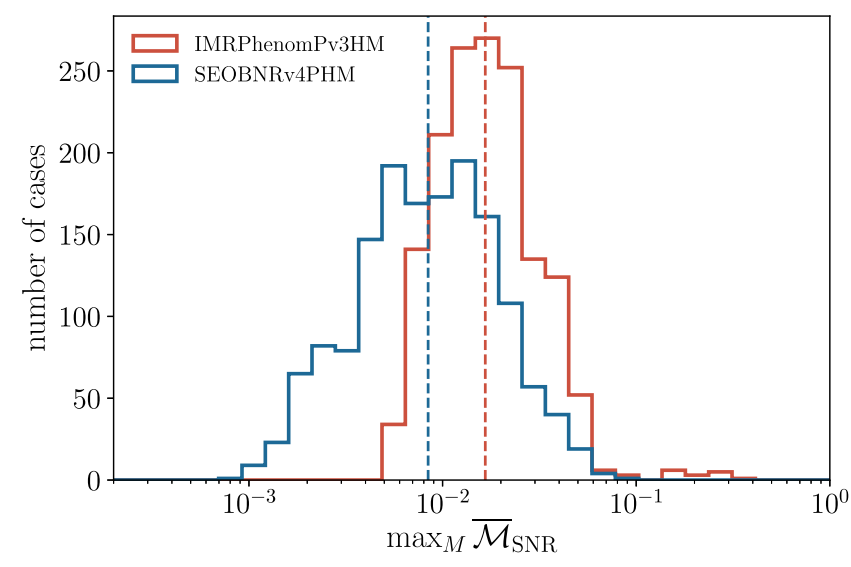

FIG. 13. The highest unfaithfulness over total mass for all cases shown in Fig. 12. The median of unfaithfulness is around $1 \%$ for SEOBNRV4PHM and 2\% for IMRPhenomPV3HM (shown as dashed vertical lines). Note that for SEOBNRV4PHM, the worst unfaithfulness is below $10 \%$ and the distribution is shifted to lower values.

the future by calibrating them to the precession sector of the SXS NR waveforms.

An interesting question is to examine the behavior of the precessing models outside the region in which their underlying aligned-spin waveforms were calibrated. To this effect we consider 1000 random cases between mass ratios $q \in[1,20]$ and spin magnitudes $\chi_{1,2} \in[0,0.99]$ and compute $\overline{\mathcal{M}}_{\mathrm{SNR}}$ between SEOBNRv4PHM and IMRPhenomPv3HM. Figure 14 shows the dependence of the unfaithfulness on the binary parameters, in particular the mass ratio, and the effective and precessing spins. We find that for mass ratios $q<8,50 \%$ of cases have unfaithfulness below $2 \%$ and $90 \%$ have unfaithfulness below $10 \%$. The unfaithfulness grows very fast with mass ratio and spin, with the highest unfaithfulness occurring at the highest mass ratio and precessing spin. This effect is enhanced due to the fact that we choose to start all the waveforms at the same frequency and for higher mass ratios, the number of cycles in band grows as $1 / \nu$ where $\nu$ is the symmetric mass ratio. These results demonstrate the importance of producing long NR simulations for large mass ratios and spins, which can be used to validate waveform models in this more extreme region of the parameter space. To design more accurate semi-analytical models in this particular region, it will be relevant to incorporate in the models the information from gravitational self-force [113-115], and also test how the choice of the underlying EOB Hamiltonians with spin effects $[116,117]$ affects the accuracy.

Finally, in Appendix A we quantify the agreement of the precessing multipolar waveform models SEOBNRV4 PHM and IMRPhenomPv3HM against the NR surrogate model NRSur7dq4 [52], which was built for binaries with mass ratios $1-4$, BH's spins up to 0.8 and binary's total masses larger than $\sim 60 M_{\odot}$. We find that the unfaithfulness between the semianalytic models and the NR surrogate largely mirrors the results of the comparison in Figs. 12 and 13. Notably, as it can be seen in Fig. 17, the unfaithfulness is generally below 3\% for both waveform families, but SEOBNRV4PHM outperforms IMRPhenomPv3HM with the former having a median at $3.3 \times 10^{-3}$, while the latter is at $1.5 \times 10^{-2}$.

\section{BAYESIAN ANALYSIS WITH MULTIPOLAR PRECESSING WAVEFORM MODELS}

We now study how the accuracy of the waveform model SEOBNRV4 PHM (and also IMRPhenomPv3HM), which we have quantified in the previous section through the unfaithfulness, affects parameter inference when synthetic signal injections are performed. To this end, we employ two mock BBH signals and do not add any detector noise to them (i.e., we work in zero noise), which is equivalent to average over many different noise realizations. This choice avoids arbitrary biases introduced by a random-noise realization,
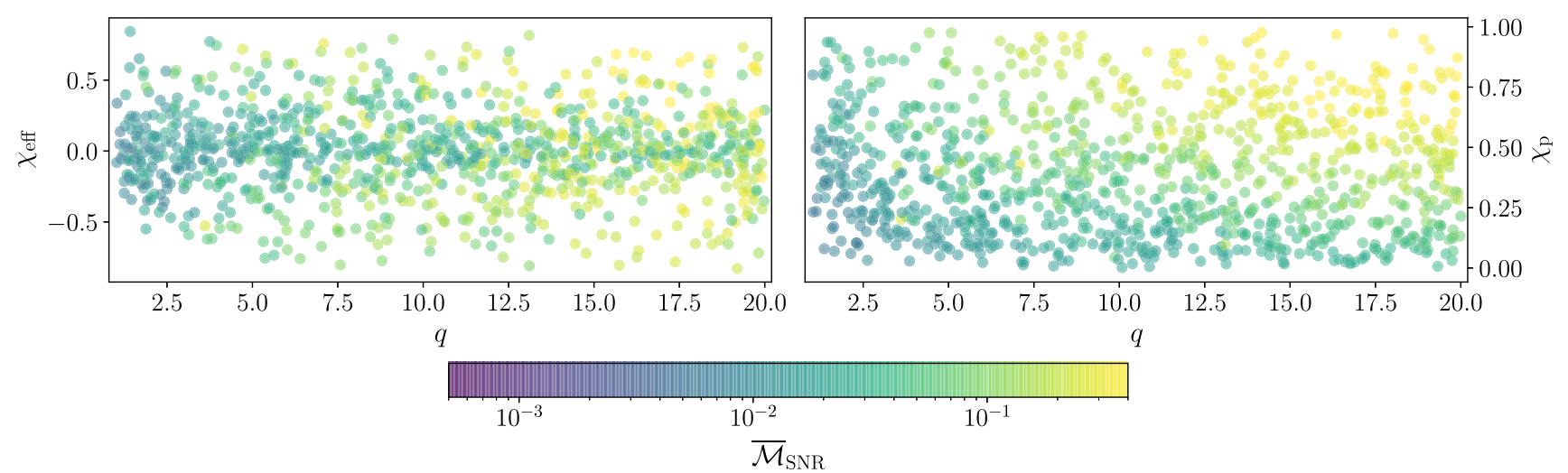

FIG. 14. Sky-and polarization-averaged unfaithfulness between SEOBNRV4PHM and IMRPhenomPV3HM for 1000 random configurations. Notice that the unfaithfulness grows both with the mass ratio and the spin and can reach very large values for $q \approx 20$ and high $\chi_{p}$. It is also clear that for cases with smaller spins the unfaithfulness remains much lower. 
and it is reasonable since the purpose of this analysis is to estimate possible biases in the binary's parameters due to inaccuracies in waveform models.

We generate the first precessing-BBH mock signal with the NRSur7dq4 model. It has mass ratio $q=3$ and a total source-frame mass of $M=70 M_{\odot}$. The spins of the two BHs are defined at a frequency of $20 \mathrm{~Hz}$, and have components $\chi_{1}=(0.30,0.00,0.50)$ and $\chi_{2}=(0.20,0.00,0.30)$. The masses and spins magnitudes ( 0.58 and 0.36 ) of this injection are compatible with those of BBH systems observed so far with LIGO and Virgo detectors [4-8]. Although the binary's parameters are not extreme, we choose the inclination with respect to the line of sight of the BBH to be $l=\pi / 3$, to emphasize the effect of higher modes. The coalescence and polarization phase, respectively $\phi$ and $\psi$, are chosen to be $1.2 \mathrm{rad}$ and $0.7 \mathrm{rad}$. The sky-position is defined by its right ascension of $0.33 \mathrm{rad}$ and its declination of $-0.6 \mathrm{rad}$ at a GPStime of $1249852257 \mathrm{~s}$. Finally, the distance to the source is set by requesting a network-SNR of 50 in the three detectors (LIGO Hanford, LIGO Livingston, and Virgo) when using the Advanced LIGO and Advanced Virgo PSD at design sensitivity [81]. The resulting distance is $800 \mathrm{Mpc}$. The unfaithfulness against this injection is $0.2 \%$ and $1 \%$ for SEOBNRV4PHM and IMRPhenomPV3HM, respectively. Although the value of the network-SNR is large for this synthetic signal, it is not excluded that the Advanced LIGO and Virgo detectors at design sensitivity could detect such loud BBH. With this study we want to test how our waveform model performs on a system with moderate precessional effect when detected with a large SNR value, considering that it has an unfaithfulness of $0.2 \%$.

For the second precessing-BBH mock signal, we use a binary with larger mass ratio and spin magnitude for the primary $\mathrm{BH}$. We employ the NR waveform SXS:BBH:0165 from the public SXS catalog having mass ratio $q=6$, and we choose the source-frame total mass $M=76 M_{\odot}$. The BH's spins, defined at a frequency of $20 \mathrm{~Hz}$, have values $\chi_{1}=(-0.06,0.78,-0.47)$ and $\chi_{2}=$ $(0.08,-0.17,-0.23)$. The BBH system in this simulation has strong spin-precession effects. We highlight that this NR waveform is one of the worst cases in term of unfaithfulness against SEOBNRV4PHM, as it is clear from Fig. 10. For this injection we choose the binary's inclination to be edge-on at $20 \mathrm{~Hz}$ to strongly emphasize higher modes. All the other binary parameters are the same of the previous injection, with the exception of the luminosity distance, which in this case is set to be $1.2 \mathrm{Gpc}$ to obtain a network-SNR of 21. The NR waveform used for this mock signal has unfaithfulness of $4.4 \%$ for SEOBNRV4 PHM and $8.8 \%$ for IMRPhenomPv3HM, thus higher than in the first injection.

For the parameter-estimation study we use the software PyCBC's pycbc_generate_hwinj [118] to prepare the mock signals, and we perform the Bayesian analysis with parallel Bilby [119], a highly parallelized version of the parameter-estimation software BILBY [120]. We choose a uniform prior in component masses in the range $[5,150] M_{\odot}$. Priors on the dimensionless spin magnitudes are uniform in [0, 0.99], while for the spin directions we use prior isotropically distributed on the unit sphere. The priors on the other parameters are the standard ones described in Appendix C.1 of Ref. [5].

We summarize in Fig. 15 the results of the parameter estimation for the first mock signal for SEOBNRV4PHM (blue), IMRPhenomPv3HM (red), and NRSur7dq4 (cyan). We report the marginalized 2D and 1D posteriors for the component masses $m_{1}$ and $m_{2}$ in the source frame (top left), the effective spin parameters $\chi_{\text {eff }}$ and $\chi_{p}$ (top right), the spin magnitude of the more massive $\mathrm{BH} a_{1}$ and its tilt angle $\theta_{1}$ (bottom left) and finally the angle $\theta_{\mathrm{JN}}$ and the luminosity distance (bottom right). In the 2D posteriors, solid contours represent $90 \%$ credible intervals and black dots show the value of the parameter used in the synthetic signal. In the 1D posteriors, they are represented respectively by dashed lines and black solid lines. As it is clear from Fig. 15, when using the waveform models SEOBNRV4PHM and NRSur7dq4, all the parameters of the synthetic signal are correctly measured within the statistical uncertainty. Moreover, the shape of the posterior distributions obtained when using SEOBNRv4PHM are similar to those recovered with NRSur7dq4 (the model used to create the synthetic signal). This means that the systematic error due to a non perfect modeling of the waveforms is negligible in this case.

For the model IMRPhenomPv3HM while masses and spins are correctly measured within the statistical uncertainty, the luminosity distance $D_{\mathrm{L}}$ and the angle $\theta_{\mathrm{JN}}$ are biased. This is consistent with the prediction obtained using Lindblom's criterion in Refs. [109,121-123]. ${ }^{9}$ In fact, according to this criterion, an unfaithfulness of $1 \%$ for IMRPhenomPv3HM would be sufficient to produce biased results at a network-SNR of 19. Thus, it is expected to observe biases when using IMRPhenomPv3HM at the network-SNR of the injection, which is 50 . In the case of SEOBNRV4PHM the unfaithfulness against the signal waveform is $0.2 \%$ and according to Lindblom's criterion we should also expect biases for network-SNRs larger than 42 , but in practice we do not observe them. We recall that Lindblom's criterion is only approximate and it has been shown in Ref. [124] to be too conservative, therefore the lack of bias that we observe is not surprising.

In Fig. 16 we summarize the results of the second mocksignal injection. The plots are the same as in Fig. 15 with the only exception that we do not have results for the

\footnotetext{
${ }^{9}$ The criterion says that a sufficient, but not necessary condition for two waveforms to become distinguishable is that the unfaithfulness $\geq\left(N_{\text {intr }}-1\right) /\left(2 \mathrm{SNR}^{2}\right)$, where $N_{\text {intr }}$ is the number of binary's intrinsic parameters, which we take to be 8 for a precessing-BBH system. Note, however, that in practice this factor can be much larger, see discussion in Ref. [124].
} 

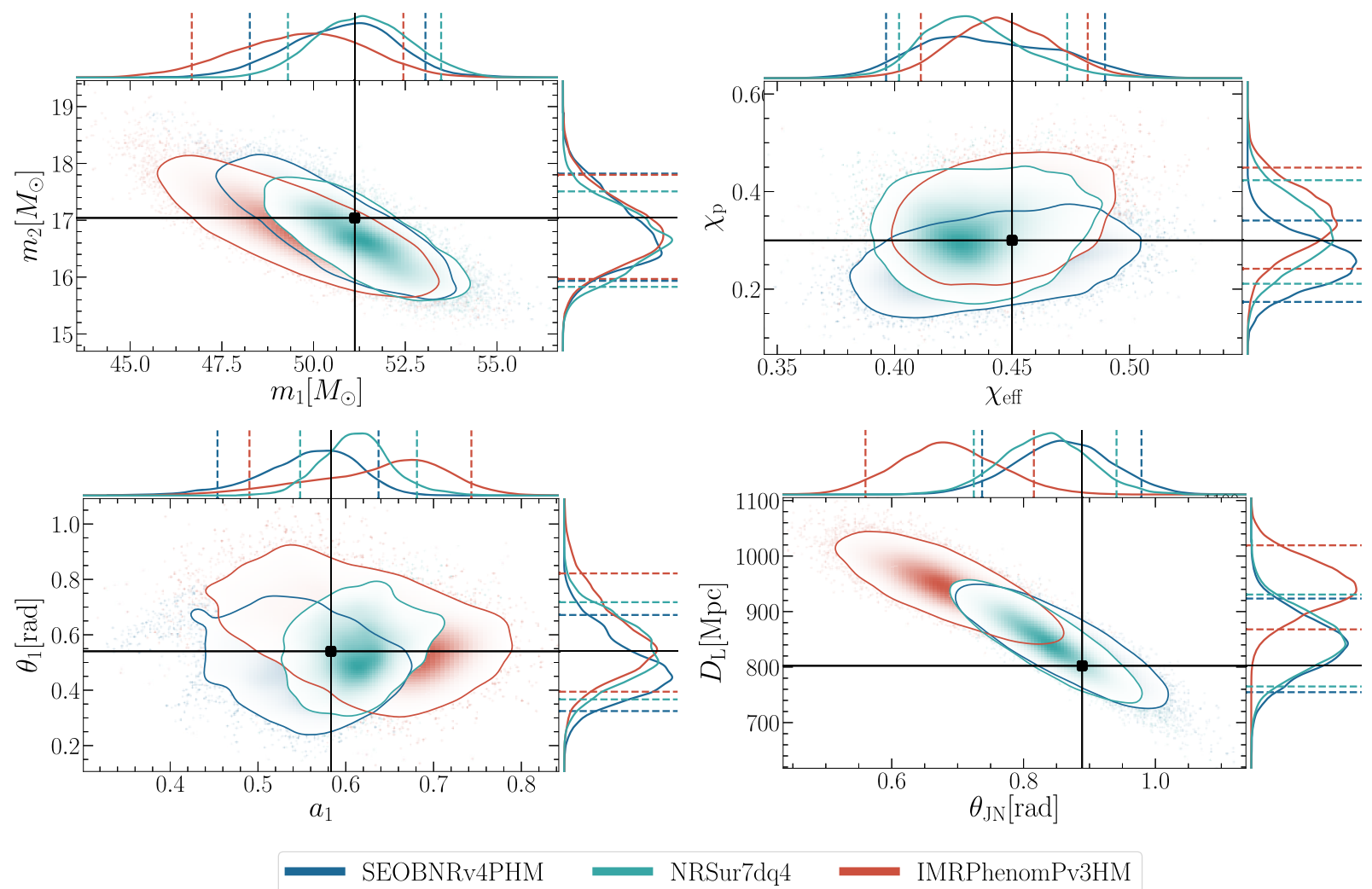

FIG. 15. 2D and 1D posterior distributions for some relevant parameters measured from the first synthetic BBH signal with mass ratio $q=3$, total source-frame mass of $M=70 M_{\odot}$, spins of the two BHs $\chi_{1}=(0.30,0.00,0.50)$ and $\chi_{2}=(0.20,0.00,0.30)$ defined at a frequency of $20 \mathrm{~Hz}$. The inclination with respect to the line of sight of the BBH is $l=\pi / 3$. The other parameters are specified in the text. The signal waveform is generated with the waveform model NRSur $7 \mathrm{dq} 4$. In the 2D posteriors, solid contours represent $90 \%$ credible intervals and black dots show the value of the parameter used in the synthetic signal. In the 1D posteriors they are represented respectively by dashed lines and black solid lines. The parameter estimation is performed with the waveform models SEOBNRV4 PHM (blue), NRSur7dq4 (cyan), and IMRPhenomPv3HM (red). Top left: component masses in the source frame, Top right: $\chi_{\text {eff }}$ and $\chi_{\mathrm{p}}$, Bottom left: magnitude and tilt angle of the primary spin, Bottom right: $\theta_{\mathrm{JN}}$ and luminosity distance.

NRSur7dq4 model since it is not available in this region of the parameter space. In this case the unfaithfulness between SEOBNRV4PHM (IMRPhenomPv3HM) and the NR waveform used for the mock signal is $4.4 \%(8.8 \%)$. According to Lindblom's criterion, at the network-SNR of this mock signal we should expect the bias due to non-perfect waveform modeling to be dominant over the statistical uncertainty for an unfaithfulness $\gtrsim 1 \%$. Therefore we might expect some biases in inferring parameters for both models. Lindblom's criterion does not say which parameters are biased and by how much. The results in Fig. 16 clearly show that both models have biases in the measurement of some parameters, but unfaithfulness of $4.4 \%$ and $8.8 \%$ induce different amount of biases and also on different set of parameters (intrinsic and extrinsic).

In particular for the component masses (top left panel of Fig. 16), the 2D posterior distribution obtained with SEOBNRV4 PHM barely include the value used for the mock signal in the $90 \%$ credible region. This measurement looks better when focusing on the 1D posterior distributions for the individual masses for which the injection values are well within the $90 \%$ credible intervals. The situation is worst for the IMRPhenomPv3 HM model, for which the 2D posterior distribution barely excludes the injection value at $90 \%$ credible level. In this case also the true value of $m_{1}$ is excluded from the $90 \%$ credible interval of the marginalized 1D posterior distribution. Furthermore, $\chi_{\text {eff }}$ and $\chi_{\mathrm{p}}$ (top right panel of Fig. 16) are correctly measured with SEOBNRV4PHM while the measurement with IMRPhenomPv3HM excludes the true value from the 2D $90 \%$ credible region. From the 1D posterior distributions it is clear that the source of this inaccuracy is the incorrect measurement of $\chi_{\mathrm{p}}$, while $\chi_{\text {eff }}$ is correctly recovered within the $90 \%$ credible interval. A similar situation is observed in the measurement of $a_{1}$ the spin magnitude of the heavier $\mathrm{BH}$ and $\theta_{1}$ its tilt angle (bottom left panel of Fig. 16). Also in this case SEOBNRv4PHM correctly measures the parameters used in the mock signal, while IMRPhenomPv3HM yields an incorrect measurement due to a bias in the estimation of $a_{1}$. Finally, we focus on the measurement of the angle $\theta_{\mathrm{JN}}$ and the luminosity distance 

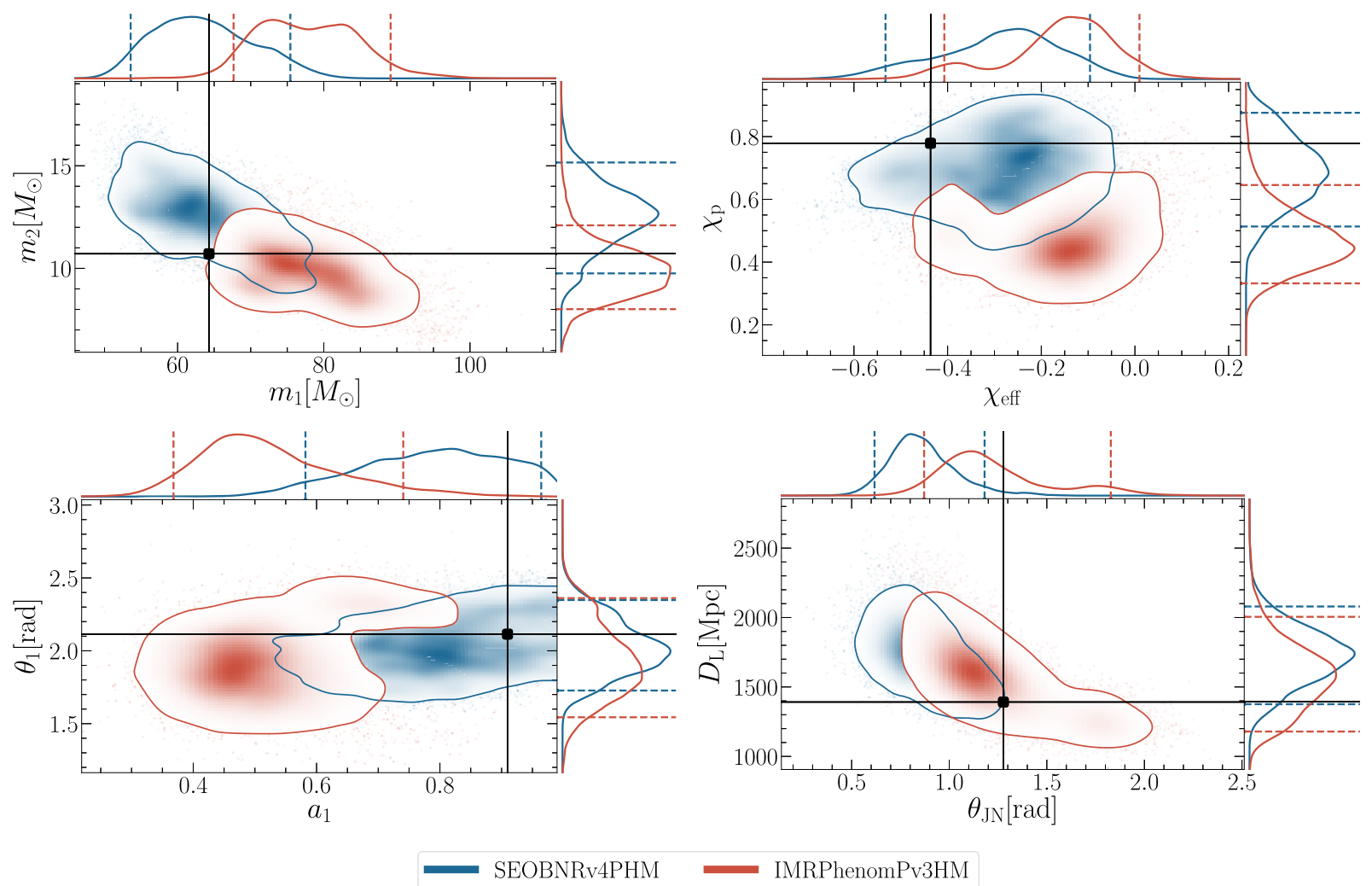

FIG. 16. 2D and 1D posterior distributions for some relevant parameters measured from the first synthetic BBH signal with mass ratio $q=6$, total source-frame mass of $M=76 M_{\odot}$, spins of the two BHs $\chi_{1}=(-0.06,0.78,-0.47)$ and $\chi_{2}=(0.08,-0.17,-0.23)$ defined at a frequency of $20 \mathrm{~Hz}$. The inclination with respect to the line of sight of the BBH is edge-on, i.e., $l=\pi / 2$. The other parameters are specified in the text. The signal waveform is generated using the NR waveform from the SXS public catalog SXS : BBH : 0165. In the $2 \mathrm{D}$ posteriors solid contours represent $90 \%$ credible intervals and black dots show the value of the parameter used in the synthetic signal. In the $1 \mathrm{D}$ posteriors they are represented respectively by dashed lines and black solid lines. The parameter estimation is performed with the waveform models SEOBNRv4 PHM (blue) and IMRPhenomPV3HM (red). Top left: component masses in the source frame, Top right: $\chi_{\text {eff }}$ and $\chi_{\mathrm{p}}$, Bottom left: magnitude and tilt angle of the primary spin, Bottom right: $\theta_{\mathrm{JN}}$ and luminosity distance.

$D_{L}$ (bottom right panel of Fig. 16). In this case the value of these parameters used in the synthetic signal is just slightly measured within the $90 \%$ credible region of the 2D posterior distribution obtained with SEOBNRV4PHM. As a consequence the luminosity distance is also barely measured within the $90 \%$ credible interval from the marginalized 1D posterior distribution and the measured value of $\theta_{\mathrm{JN}}$ results outside the $90 \%$ credible interval of the 1D posterior distribution. The posterior distributions obtained using IMRPhenomPv3HM are instead correctly measuring the parameters of the mock signal. We can conclude that even with an unfaithfulness of $4.4 \%$ against the NR waveform used for the mock signal the SEOBNRV4 PHM model is able to correctly measure most of the binary parameters, notably the intrinsic ones, such as masses and spins.

\section{CONCLUSIONS}

In this paper we have developed and validated the first inspiral-merger-ringdown precessing waveform model in the EOB approach, SEOBNRv4 PHM, that includes multipoles beyond the dominant quadrupole.

Following previous precessing SEOBNR models $[26,33,86]$, we have built such a model twisting up the aligned-spin waveforms of SEOBNRV4HM $[45,46]$ from the coprecessing $[21,23,42-44]$ to the inertial frame, through the EOB equations of motion for the spins and orbital angular momentum. With respect to the previous precessing SEOBNR model, SEOBNRV3P [33], which has been used in LIGO/Virgo data analysis $[5,59,60]$, the new model (i) employs a more accurate aligned-spin two-body dynamics, since, in the nonprecessing limit, it reduces to SEOBNRV4HM, which was calibrated to 157 SXS NR simulations [47,48], and 13 waveforms [49] from BH perturbation theory, (ii) includes in the coprecessing frame the modes $(2, \pm 2),(2, \pm 1),(3, \pm 3),(4, \pm 4)$ and $(5, \pm 5)$, instead of only $(2, \pm 2),(2, \pm 1)$, (iii) incorporates the merger-ringdown signal in the coprecessing frame instead of the inertial frame, (iv) describes the merger-ringdown stage through a phenomenological fit to NR waveforms 
[45,46], and (v) uses more accurate NR fits for the final spin of the remnant $\mathrm{BH}$.

The improvement in accuracy between SEOBNRv4 and SEOBNRV3 P (i.e., the models with only the $\ell=2$ modes) is evident from Fig. 8, where we have compared those models to the public SXS catalog of 1405 precessing NR waveforms, and the new 118 SXS NR waveforms produced for this work. The impact of including higher modes in semianalytical models to achieve higher accuracy to multipolar NR waveforms is demonstrated in Fig. 9. Figures 10, 11,12 and 14 quantify the comparison of the multipolar precessing SEOBNRV4 PHM and IMRPhenomPV3HM to all SXS NR precessing waveforms at our disposal. We have found that for the SEOBNRV4 PHM model, 94\% (57\%) of the cases have maximum unfaithfulness value, in the total mass range $20-200 M_{\odot}$, below $3 \%(1 \%)$. Those numbers change to $83 \%$ (20\%) when using the IMRPhenomPv3HM. We have found several cases with large unfaithfulness ( $>10 \%$ ) for IMRPhenomPv3HM, coming from a region of parameter space with $q \gtrsim 4$ and large $(\simeq 0.8)$ spins antialigned with the orbital angular momentum, which appear to be connected to unphysical features in the underlying precession model, and cause unusual oscillations in the waveform's amplitude and phase. The better accuracy of SEOBNRV4PHM with respect to IMRPhenomPV3HM is also confirmed by the comparisons with the NR surrogate model NRSur7dq4, as shown in Fig. 17. We have investigated in which region of the parameter space the unfaithfulness against NR waveforms and NRSur7dq4 lies, and have found, not surprisingly, that it occurs where both mass ratios and spins are large (see Fig. 18). When comparing SEOBNRV4PHM and IMRPhenomPv3HM outside the region in which the aligned-spin underlying model was calibrated, we have also found that the largest differences reside when mass ratios are larger than 4 and spins larger than 0.8 (see Fig. 14). To improve the accuracy of the models in those more challenging regions, we would need NR simulations, but also more information from analytical methods, such as the gravitational self-force [113-115], and resummed EOB Hamiltonians with spins [116,117].

To quantify how the modeling inaccuracy, estimated by the unfaithfulness, impacts the inference of binary's parameters, we have performed two parameter-estimation studies using Bayesian analysis. Working with the Advanced LIGO and Virgo network at design sensitivity, we have injected in zero noise two precessing-BBH mock signals with mass ratio 3 and 6 , having SNR of 50 and 21, with inclination of $\pi / 3$ and $\pi / 2$ with respect to the line of sight respectively, and recovered them with SEOBNRV4 PHM and IMRPhenomPv3HM. The unfaithfulness values of those models against the synthetic signals considered (i.e., NRSurd7q4 and SXS:BBH:0165) range from $0.2 \%$ to $8.8 \%$. The results are summarized in Figs. 15 and 16. Overall, we have found that Lindblom's criterion [109,
121-124] is too conservative and predicts visible biases at SNRs lower than what we have obtained through the Bayesian analysis. In particular, we have found, when doing inference with SEOBNRV4PHM, that an unfaithfulness of $0.2 \%$ may produce no biases up to SNR of 50, while an unfaithfulness of $2.2 \%$ can produce biases only for some extrinsic parameters, such as distance and inclination, but not for binary's masses and spins at SNR of 21. A more comprehensive Bayesian study will be needed to quantify, in a more realistic manner, the modeling systematics of SEOBNRV4 PHM, if this model were used during the fourth observation run of Avanced LIGO and Virgo in 2022 (i.e., the run at design sensitivity).

The newly produced 118 SXS NR waveforms extend the coverage of binary's parameter space, spanning mass ratios $q=1-4$, (dimensionless) spins $\chi_{1,2}=0.3-0.9$, and different orientations to maximize the number of precessional cycles. As we have emphasized, the waveform model SEOBNRV4HM is not calibrated to NR waveforms in the precessing sector, only the aligned-spin sector was calibrated in Refs. [45,46]. Despite this, the accuracy of the model is very good, and it can be further improved in the future if we calibrate the model to the 1404 plus 118 SXS NR precessing waveforms at our disposal. This will be an important goal for the upcoming LIGO and Virgo O4 run in early 2022. Furthermore, SEOBNRV4 HM assumes the following symmetry among modes $h_{\ell m}=(-1)^{\ell} h_{\ell-m}^{*}$ in the coprecessing frame, which however no longer holds in presence of precession. As discussed in Sec. II D, forcing this assumption causes unfaithfulness on the order of a few percent. Thus, to achieve better accuracy, when calibrating the model to NR waveforms, the mode-symmetry would need to be relaxed.

Finally, SEOBNRV4HM uses PN-resumed factorized modes that were developed for aligned-spin BBHs $[98,99]$, thus they neglect the projection of the spins on the orbital plane. To obtain high-precision waveform models, it will be relevant to extend the factorized modes to precession. Considering the variety of GW signals that the improved sensitivity of LIGO and Virgo detectors is allowing to observe, it will also be important to include in the multipolar SEOBNR waveform models the more challenging $(3,2)$ and $(4,3)$ modes, which are characterized my mode mixing [125-127]. Their contribution is no longer negligible for high total-mass and/or large massratio binaries, especially if observed away from face-on (face-off).

Lastly, being a time-domain waveform model generated by solving ordinary differential equations, SEOBNRV $4 \mathrm{HM}$ is not a fast waveform model, especially for low total-mass binaries. To speed up the waveform generation, a reducedorder modeling version has been recently developed [128]. Alternative methods that employ a fast evolution of the EOB Hamilton equations in the postadiabatic approximation during the long inspiral phase have been suggested 
[129], and we are currently implementing them in the simpler nonprecessing limit in LAL.

\section{ACKNOWLEDGMENTS}

It is our pleasure to thank Andrew Matas for providing us with the scripts to make the parameter-estimation plots, and Sebastian Khan for useful discussions on the faithfulness calculation. We would also like to thank the SXS collaboration for help and support with the SPEC code in producing the new NR simulations presented in this paper, and for making the large catalog of $\mathrm{BBH}$ simulations publicly available. R. H. acknowledges support through OAC-1550514, OAC-2004879, and ACI-1238993. The new 118 SXS NR simulations were produced using the high-performance compute (HPC) cluster Minerva at the Max Planck Institute for Gravitational Physics in Potsdam, on the Hydra cluster at the Max Planck

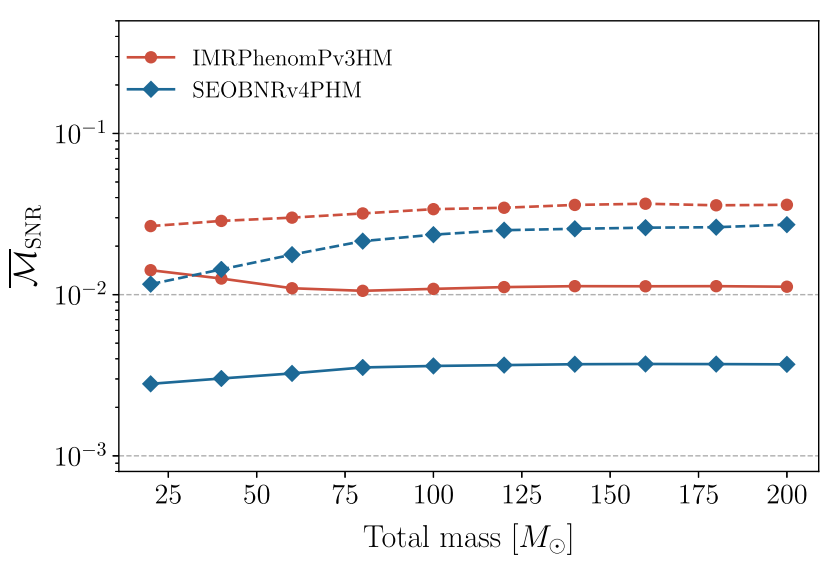

Society at the Garching facility, and on the SciNet cluster at the University of Toronto. The data-analysis studies were obtained with the HPC clusters Hypatia and Minerva at the Max Planck Institute for Gravitational Physics. The transformation and manipulation of waveforms were done using the GWFrames package $[83,130]$.

\section{APPENDIX A: COMPARISON OF MULTIPOLAR PRECESSING MODELS TO NUMERICAL- RELATIVITY SURROGATE WAVEFORMS}

In this Appendix we compare directly SEOBNRv4 PHM and IMRPhenomPv3HM to the NR surrogate model NRSur7dq4. We choose a starting frequency corresponding to $20 \mathrm{~Hz}$ at $70 M_{\odot}$ (this is essentially the limit of the length for NR surrogate waveforms). We generate 1000 random configurations, uniform in mass ratio $q \in$ $[1,4]$ and in spin magnitudes $\in[0,0.8]$, and with random

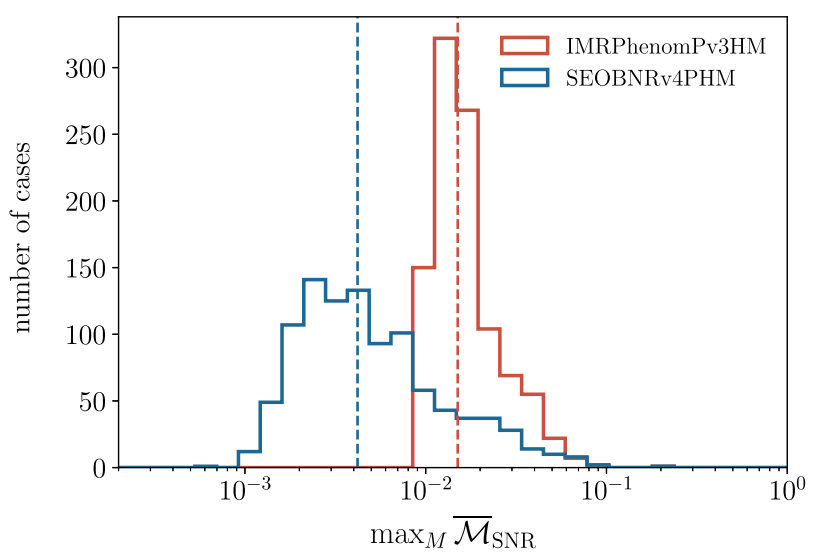

FIG. 17. The summary of the sky-and-polarization averaged, SNR-weighted unfaithfulness as a function of binary's total mass for inclination $\imath=\pi / 3$, among the NRSur7dq4 model and the IMRPhenomPv3HM and SEOBNRV4 PHM models. Left: The solid (dashed) lines show the median (95th percentile) as a function of total mass, cf Fig. 12. Right: maximum unfaithfulness over all total masses, cf. Fig. 13. The unfaithfulness is low using both waveform families, however, SEOBNRV4P (HM) has lower median unfaithfulness by a factor of 4.3 .

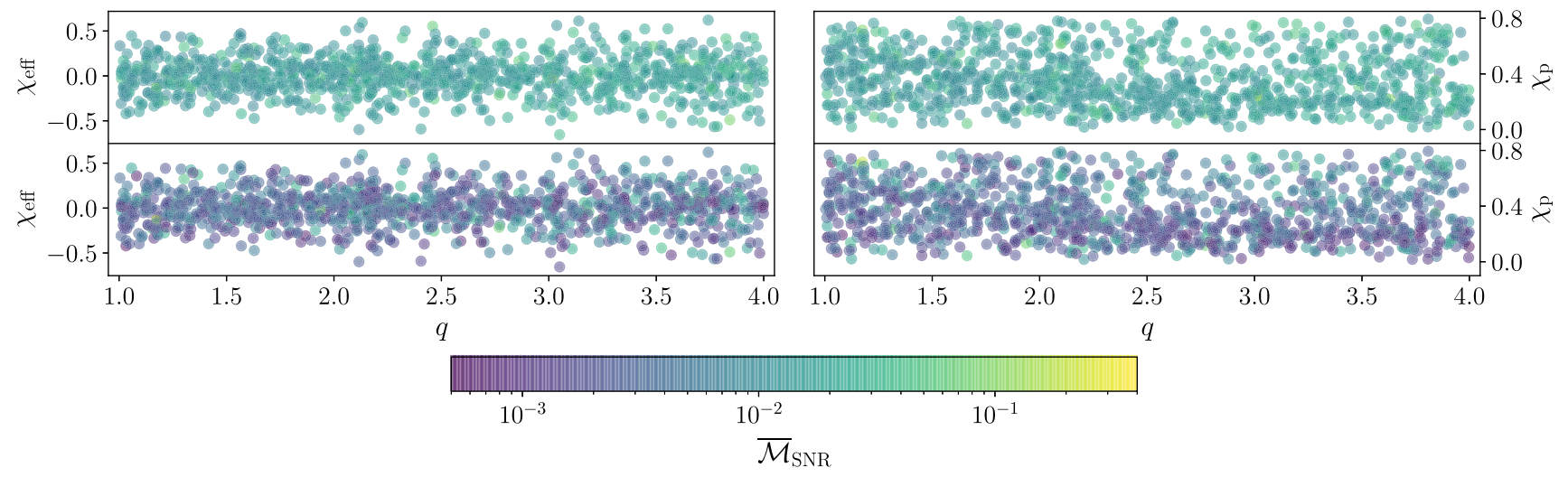

FIG. 18. The maximum sky-and-polarization averaged, SNR-weighted unfaithfulness as a function of binary's total mass for inclination $\imath=\pi / 3$, between the models IMRPhenomPv3HM (top) and SEOBNRv4PHM (bottom), and the NR surrogate, cf. Fig. 14. The unfaithfulness is strongly dependent on the intrinsic parameters, especially the spins. 
directions uniform on the unit sphere. The left panel of Fig. 17 shows the summary of the unfaithfulness as a function of total mass for all the cases considered, for IMRPhenomPV3HM and SEOBNRV4 PHM. We see that the median and 95th percentile values for both models are close to the values in Fig. 12, with SEOBNRv4 PHM having a median unfaithfulness below $1 \%$ and IMRPhenomPv3HM about a factor of 3 larger. The right panel of Fig. 17 shows the maximum unfaithfulness distribution and the same trends are also observed. SEOBNRV4PHM outperforms IMRPhenomPv3HM, with the median of the former being 4 times smaller than the one of the latter. Finally, to gain further insight into the behavior of the waveform models across the parameter space, we show in Fig. 18 the maximum unfaithfulness as a function of mass ratio and the effective spin.

\section{APPENDIX B: PARAMETERS OF THE NEW 118 NR SIMULATIONS}

TABLE II. The parameters of the runs in the new precessing catalog. Note that all the parameters are provided at the relaxed time and in the LAL source frame [131].

\begin{tabular}{|c|c|c|c|c|c|}
\hline ID & $q$ & $\chi_{1}$ & $\chi_{2}$ & $M \Omega$ & \# of orbits \\
\hline PrecBBH000001 & 1.2499 & $(-0.272,-0.628,0.414)$ & $(-0.212,-0.653,0.41)$ & 0.01632 & 21 \\
\hline PrecBBH000002 & 1.2500 & $(-0.629,0.202,0.451)$ & $(-0.13,-0.708,0.348)$ & 0.01645 & 20 \\
\hline PrecBBH000003 & 1.2499 & $(0.68,-0.104,0.408)$ & $(0.71,-0.153,-0.335)$ & 0.01616 & 19 \\
\hline PrecBBH000004 & 1.2501 & $(0.309,-0.593,0.439)$ & $(0.611,0.325,-0.401)$ & 0.01627 & 18 \\
\hline PrecBBH000005 & 1.2500 & $(0.269,-0.684,-0.317)$ & $(0.393,-0.57,0.4)$ & 0.01626 & 18 \\
\hline PrecBBH000006 & 1.2500 & $(0.561,-0.488,-0.293)$ & $(0.37,0.611,0.361)$ & 0.01623 & 18 \\
\hline PrecBBH000007 & 1.2499 & $(-0.671,0.287,-0.328)$ & $(-0.694,0.205,-0.339)$ & 0.01651 & 16 \\
\hline PrecBBH000008 & 1.2501 & $(-0.7,0.269,-0.277)$ & $(-0.133,-0.669,-0.418)$ & 0.01653 & 16 \\
\hline PrecBBH000009 & 2.4998 & $(0.279,0.579,0.387)$ & $(0.138,0.631,0.381)$ & 0.01604 & 24 \\
\hline PrecBBH000010 & 2.5000 & $(-0.577,0.26,0.403)$ & $(-0.021,-0.679,0.317)$ & 0.01633 & 24 \\
\hline PrecBBH000011 & 2.4999 & $(-0.604,0.23,0.381)$ & $(-0.608,0.096,-0.428)$ & 0.01631 & 23 \\
\hline PrecBBH000012 & 2.4998 & $(-0.587,0.238,0.402)$ & $(-0.014,-0.576,-0.48)$ & 0.01630 & 23 \\
\hline PrecBBH000013 & 2.4998 & $(-0.531,0.349,-0.399)$ & $(-0.65,-0.043,0.371)$ & 0.01636 & 19 \\
\hline PrecBBH000014 & 2.4998 & $(-0.554,0.332,-0.382)$ & $(0.012,-0.683,0.309)$ & 0.01638 & 19 \\
\hline PrecBBH000015 & 2.4998 & $(0.052,0.633,-0.399)$ & $(-0.096,0.62,-0.411)$ & 0.01605 & 18 \\
\hline PrecBBH000016 & 2.4997 & $(0.615,0.166,-0.396)$ & $(-0.326,0.497,-0.457)$ & 0.01606 & 18 \\
\hline PrecBBH000017 & 3.4997 & $(0.421,0.298,0.306)$ & $(0.301,0.417,0.309)$ & 0.01598 & 27 \\
\hline PrecBBH000018 & 3.4992 & $(0.464,0.218,0.312)$ & $(-0.348,0.402,0.277)$ & 0.01599 & 27 \\
\hline PrecBBH000019 & 3.4996 & $(0.242,0.455,0.307)$ & $(0.127,0.471,-0.349)$ & 0.01598 & 26 \\
\hline PrecBBH000020 & 3.4999 & $(0.514,-0.006,0.31)$ & $(-0.139,0.451,-0.371)$ & 0.01602 & 26 \\
\hline PrecBBH000021 & 3.4993 & $(-0.4,0.297,-0.335)$ & $(-0.518,-0.054,0.298)$ & 0.01631 & 22 \\
\hline PrecBBH000022 & 3.4995 & $(0.464,0.18,-0.335)$ & $(-0.358,0.395,0.275)$ & 0.01605 & 22 \\
\hline PrecBBH000023 & 3.4991 & $(0.414,-0.273,-0.338)$ & $(0.472,-0.09,-0.358)$ & 0.01606 & 21 \\
\hline PrecBBH000024 & 3.4997 & $(0.256,-0.431,-0.329)$ & $(0.225,0.401,-0.385)$ & 0.01609 & 21 \\
\hline PrecBBH000025 & 1.2501 & $(-0.661,0.193,0.407)$ & $(0.0,-0.0,0.0)$ & 0.01645 & 19 \\
\hline PrecBBH000026 & 1.2501 & $(-0.466,-0.618,-0.2)$ & $(0.0,-0.0,0.0)$ & 0.01638 & 17 \\
\hline PrecBBH000027 & 2.4999 & $(0.099,0.637,0.383)$ & $(0.0,-0.0,0.0)$ & 0.01601 & 23 \\
\hline PrecBBH000028 & 2.5003 & $(0.557,0.357,-0.354)$ & $(0.0,0.0,-0.0)$ & 0.01609 & 19 \\
\hline PrecBBH000029 & 3.5006 & $(0.458,-0.242,0.302)$ & $(0.0,0.0,0.0)$ & 0.01603 & 27 \\
\hline PrecBBH000030 & 3.4996 & $(-0.397,-0.32,-0.316)$ & $(0.0,-0.0,0.0)$ & 0.01619 & 22 \\
\hline PrecBBH000031 & 1.0001 & $(-0.752,0.179,0.461)$ & $(-0.0,-0.0,0.0)$ & 0.01646 & 19 \\
\hline PrecBBH000032 & 1.0002 & $(-0.836,0.259,-0.206)$ & $(-0.0,-0.0,0.0)$ & 0.01649 & 17 \\
\hline PrecBBH000033 & 2.0000 & $(-0.709,0.269,0.445)$ & $(-0.0,-0.0,0.0)$ & 0.01638 & 22 \\
\hline PrecBBH000034 & 2.0004 & $(0.027,0.793,-0.379)$ & $(-0.0,0.0,-0.0)$ & 0.01605 & 18 \\
\hline PrecBBH000035 & 3.2002 & $(0.681,0.112,0.405)$ & $(0.0,0.0,0.0)$ & 0.01599 & 26 \\
\hline PrecBBH000036 & 3.1995 & $(0.162,0.597,-0.507)$ & $(-0.0,-0.0,0.0)$ & 0.01600 & 20 \\
\hline PrecBBH000037 & 3.9994 & $(0.596,-0.106,0.352)$ & $(-0.0,-0.0,-0.0)$ & 0.01598 & 29 \\
\hline PrecBBH000038 & 4.0003 & $(-0.146,-0.481,-0.487)$ & $(-0.0,0.0,-0.0)$ & 0.01613 & 22 \\
\hline PrecBBH000039 & 1.0000 & $(-0.542,0.137,0.332)$ & $(-0.0,-0.0,-0.0)$ & 0.01646 & 19 \\
\hline
\end{tabular}


TABLE II. (Continued)

\begin{tabular}{|c|c|c|c|c|c|}
\hline ID & $q$ & $\chi_{1}$ & $\chi_{2}$ & $M \Omega$ & \# of orbits \\
\hline PrecBBH000040 & 1.0000 & $(-0.614,0.183,-0.108)$ & $(-0.0,-0.0,-0.0)$ & 0.01649 & 17 \\
\hline PrecBBH000041 & 2.0001 & $(-0.48,0.195,0.303)$ & $(0.0,-0.0,0.0)$ & 0.01639 & 21 \\
\hline PrecBBH000042 & 2.0003 & $(-0.509,0.261,-0.181)$ & $(0.0,-0.0,-0.0)$ & 0.01644 & 19 \\
\hline PrecBBH000043 & 2.5002 & $(0.349,0.252,0.254)$ & $(0.0,0.0,-0.0)$ & 0.01606 & 22 \\
\hline PrecBBH000044 & 2.5000 & $(0.456,0.13,-0.158)$ & $(-0.0,-0.0,-0.0)$ & 0.01607 & 20 \\
\hline PrecBBH000045 & 3.9999 & $(-0.265,0.146,0.176)$ & $(0.0,0.0,-0.0)$ & 0.01621 & 27 \\
\hline PrecBBH000046 & 4.0003 & $(0.25,-0.213,-0.122)$ & $(-0.0,-0.0,-0.0)$ & 0.01603 & 25 \\
\hline PrecBBH000047 & 2.9997 & $(0.249,0.072,0.152)$ & $(0.0,-0.0,-0.0)$ & 0.01604 & 23 \\
\hline PrecBBH000048 & 3.0000 & $(0.228,-0.183,-0.067)$ & $(-0.0,-0.0,-0.0)$ & 0.01610 & 22 \\
\hline PrecBBH000050 & 1.0001 & $(-0.709,0.187,0.522)$ & $(-0.18,-0.79,0.391)$ & 0.01644 & 21 \\
\hline PrecBBH000051 & 1.0000 & $(-0.768,0.118,0.453)$ & $(-0.747,0.299,-0.402)$ & 0.01646 & 18 \\
\hline PrecBBH000053 & 1.0000 & $(-0.747,0.299,-0.402)$ & $(-0.768,0.117,0.453)$ & 0.01646 & 18 \\
\hline PrecBBH000054 & 1.0001 & $(-0.79,0.265,-0.339)$ & $(-0.161,-0.801,0.377)$ & 0.01648 & 18 \\
\hline PrecBBH000055 & 1.0000 & $(-0.748,0.286,-0.41)$ & $(-0.748,0.286,-0.41)$ & 0.01651 & 16 \\
\hline PrecBBH000056 & 1.0001 & $(-0.791,0.266,-0.335)$ & $(-0.207,-0.71,-0.514)$ & 0.01655 & 15 \\
\hline PrecBBH000057 & 1.9997 & $(-0.715,0.242,0.452)$ & $(-0.757,0.023,0.448)$ & 0.01634 & 23 \\
\hline PrecBBH000058 & 2.0000 & $(-0.681,0.276,0.484)$ & $(-0.061,-0.797,0.368)$ & 0.01636 & 23 \\
\hline PrecBBH000059 & 1.9997 & $(-0.725,0.222,0.447)$ & $(-0.706,0.16,-0.499)$ & 0.01634 & 21 \\
\hline PrecBBH000060 & 2.0001 & $(-0.695,0.242,0.482)$ & $(-0.059,-0.655,-0.584)$ & 0.01636 & 21 \\
\hline PrecBBH000061 & 1.9998 & $(0.674,0.294,-0.483)$ & $(0.529,0.539,0.451)$ & 0.01611 & 18 \\
\hline PrecBBH000062 & 1.9999 & $(-0.441,-0.618,-0.444)$ & $(0.762,-0.218,0.381)$ & 0.01632 & 18 \\
\hline PrecBBH000063 & 1.9998 & $(-0.628,0.392,-0.475)$ & $(-0.7,0.137,-0.514)$ & 0.01643 & 16 \\
\hline PrecBBH000064 & 2.0000 & $(-0.188,0.727,-0.458)$ & $(-0.608,-0.3,-0.561)$ & 0.01604 & 16 \\
\hline PrecBBH000065 & 3.1997 & $(-0.633,0.268,0.409)$ & $(-0.689,-0.046,0.403)$ & 0.01622 & 27 \\
\hline PrecBBH000066 & 3.1998 & $(-0.611,0.292,0.426)$ & $(0.012,-0.728,0.331)$ & 0.01623 & 27 \\
\hline PrecBBH000067 & 3.1996 & $(0.606,0.327,0.408)$ & $(0.436,0.335,-0.581)$ & 0.01598 & 26 \\
\hline PrecBBH000068 & 3.1998 & $(-0.624,0.27,0.421)$ & $(0.018,-0.487,-0.634)$ & 0.01623 & 25 \\
\hline PrecBBH000069 & 3.1995 & $(-0.444,0.373,-0.551)$ & $(-0.692,-0.085,0.391)$ & 0.01634 & 20 \\
\hline PrecBBH000070 & 3.1992 & $(-0.51,-0.29,-0.544)$ & $(0.632,-0.342,0.35)$ & 0.01627 & 20 \\
\hline PrecBBH000071 & 3.1991 & $(0.4,0.409,-0.559)$ & $(0.228,0.504,-0.577)$ & 0.01611 & 18 \\
\hline PrecBBH000072 & 3.1994 & $(0.245,0.527,-0.549)$ & $(-0.51,0.053,-0.613)$ & 0.01600 & 18 \\
\hline PrecBBH000073 & 4.0002 & $(0.604,0.004,0.354)$ & $(0.559,0.214,0.363)$ & 0.01597 & 30 \\
\hline PrecBBH000074 & 3.9992 & $(-0.004,-0.595,0.369)$ & $(0.573,0.241,0.322)$ & 0.01607 & 30 \\
\hline PrecBBH000075 & 4.0000 & $(-0.549,0.252,0.354)$ & $(-0.441,0.048,-0.542)$ & 0.01616 & 28 \\
\hline PrecBBH000076 & 3.9993 & $(-0.538,0.262,0.363)$ & $(0.034,-0.402,-0.572)$ & 0.01618 & 28 \\
\hline PrecBBH000077 & 4.0003 & $(-0.361,0.309,-0.513)$ & $(-0.6,-0.101,0.345)$ & 0.01623 & 22 \\
\hline PrecBBH000078 & 4.0001 & $(0.466,0.089,-0.515)$ & $(-0.366,0.503,0.321)$ & 0.01604 & 22 \\
\hline PrecBBH000079 & 4.0003 & $(-0.435,0.179,-0.518)$ & $(-0.416,-0.118,-0.55)$ & 0.01624 & 21 \\
\hline PrecBBH000080 & 4.0000 & $(0.139,0.456,-0.513)$ & $(-0.422,-0.03,-0.557)$ & 0.01599 & 21 \\
\hline PrecBBH000081 & 1.0000 & $(-0.545,0.12,0.333)$ & $(-0.545,0.12,0.333)$ & 0.01643 & 20 \\
\hline PrecBBH000082 & 1.0000 & $(-0.519,0.141,0.365)$ & $(-0.132,-0.565,0.293)$ & 0.01645 & 20 \\
\hline PrecBBH000083 & 1.0000 & $(-0.549,0.107,0.33)$ & $(-0.581,0.198,-0.213)$ & 0.01646 & 18 \\
\hline PrecBBH000084 & 1.0000 & $(-0.52,0.126,0.369)$ & $(-0.163,-0.574,-0.258)$ & 0.01648 & 18 \\
\hline PrecBBH000085 & 1.0000 & $(-0.582,0.197,-0.213)$ & $(-0.55,0.106,0.33)$ & 0.01646 & 18 \\
\hline PrecBBH000086 & 1.0000 & $(-0.596,0.188,-0.176)$ & $(-0.123,-0.57,0.286)$ & 0.01648 & 18 \\
\hline PrecBBH000087 & 1.0000 & $(-0.582,0.192,-0.215)$ & $(-0.582,0.192,-0.215)$ & 0.01650 & 17 \\
\hline PrecBBH000088 & 1.0000 & $(-0.6,0.181,-0.172)$ & $(-0.151,-0.573,-0.266)$ & 0.01651 & 16 \\
\hline PrecBBH000089 & 1.9999 & $(0.513,-0.058,0.305)$ & $(0.511,0.046,0.311)$ & 0.01615 & 22 \\
\hline PrecBBH000090 & 2.0003 & $(-0.467,0.197,0.322)$ & $(-0.039,-0.537,0.264)$ & 0.01638 & 22 \\
\hline PrecBBH000091 & 2.0001 & $(0.278,0.433,0.308)$ & $(0.238,0.5,-0.231)$ & 0.01604 & 21 \\
\hline PrecBBH000092 & 1.9999 & $(-0.47,0.186,0.323)$ & $(-0.041,-0.533,-0.273)$ & 0.01638 & 21 \\
\hline PrecBBH000093 & 1.9999 & $(-0.495,0.257,-0.221)$ & $(-0.518,0.005,0.302)$ & 0.01639 & 19 \\
\hline PrecBBH000094 & 1.9999 & $(0.553,-0.092,-0.214)$ & $(-0.063,0.531,0.272)$ & 0.01612 & 19 \\
\hline PrecBBH000095 & 1.9999 & $(-0.494,0.258,-0.221)$ & $(-0.544,0.074,-0.242)$ & 0.01641 & 18 \\
\hline PrecBBH000096 & 1.9998 & $(-0.532,-0.185,-0.206)$ & $(0.332,-0.419,-0.273)$ & 0.01637 & 18 \\
\hline
\end{tabular}


TABLE II. (Continued)

\begin{tabular}{|c|c|c|c|c|c|}
\hline ID & $q$ & $\chi_{1}$ & $\chi_{2}$ & $M \Omega$ & $\#$ of orbits \\
\hline PrecBBH000097 & 2.4999 & $(0.003,0.43,0.255)$ & $(-0.085,0.423,0.252)$ & 0.01603 & 23 \\
\hline PrecBBH000098 & 2.5000 & $(0.075,0.421,0.26)$ & $(-0.435,0.003,0.246)$ & 0.01604 & 23 \\
\hline PrecBBH000099 & 2.5000 & $(0.128,0.41,0.256)$ & $(0.057,0.461,-0.184)$ & 0.01601 & 22 \\
\hline PrecBBH000100 & 2.4999 & $(-0.06,0.424,0.259)$ & $(-0.435,-0.135,-0.206)$ & 0.01601 & 22 \\
\hline PrecBBH000101 & 2.5002 & $(0.195,-0.426,-0.176)$ & $(0.318,-0.294,0.25)$ & 0.01616 & 20 \\
\hline PrecBBH000102 & 2.5001 & $(0.468,-0.022,-0.175)$ & $(-0.121,0.428,0.229)$ & 0.01610 & 20 \\
\hline PrecBBH000103 & 2.5002 & $(0.456,-0.095,-0.182)$ & $(0.459,0.038,-0.194)$ & 0.01610 & 20 \\
\hline PrecBBH000104 & 2.5000 & $(0.293,0.366,-0.173)$ & $(-0.41,0.199,-0.206)$ & 0.01610 & 19 \\
\hline PrecBBH000105 & 4.0011 & $(-0.302,0.006,0.177)$ & $(-0.256,-0.156,0.18)$ & 0.01616 & 28 \\
\hline PrecBBH000106 & 4.0001 & $(0.251,0.165,0.179)$ & $(-0.236,0.197,0.168)$ & 0.01596 & 28 \\
\hline PrecBBH000107 & 4.0008 & $(0.252,0.166,0.177)$ & $(0.198,0.253,-0.138)$ & 0.01598 & 27 \\
\hline PrecBBH000108 & 3.9994 & $(0.038,-0.298,0.18)$ & $(0.267,0.17,-0.15)$ & 0.01608 & 27 \\
\hline PrecBBH000109 & 4.0002 & $(0.171,0.277,-0.129)$ & $(0.048,0.298,0.177)$ & 0.01592 & 25 \\
\hline PrecBBH000110 & 4.0005 & $(-0.122,-0.303,-0.125)$ & $(0.305,0.038,0.168)$ & 0.01611 & 25 \\
\hline PrecBBH000111 & 3.9999 & $(0.278,0.168,-0.13)$ & $(0.193,0.257,-0.138)$ & 0.01597 & 24 \\
\hline PrecBBH000112 & 4.0001 & $(0.249,0.21,-0.128)$ & $(-0.274,0.165,-0.143)$ & 0.01598 & 24 \\
\hline PrecBBH000113 & 3.0002 & $(-0.232,0.115,0.152)$ & $(-0.257,-0.004,0.154)$ & 0.01627 & 24 \\
\hline PrecBBH000114 & 3.0001 & $(0.001,-0.257,0.154)$ & $(0.243,0.096,0.148)$ & 0.01614 & 24 \\
\hline PrecBBH000115 & 3.0000 & $(0.123,0.227,0.153)$ & $(0.069,0.281,-0.078)$ & 0.01601 & 23 \\
\hline PrecBBH000116 & 2.9997 & $(0.236,0.102,0.154)$ & $(-0.179,0.226,-0.083)$ & 0.01615 & 23 \\
\hline PrecBBH000117 & 2.9999 & $(-0.252,0.144,-0.074)$ & $(-0.258,-0.009,0.153)$ & 0.01629 & 22 \\
\hline PrecBBH000118 & 3.0001 & $(0.163,-0.242,-0.071)$ & $(0.14,0.222,0.144)$ & 0.01611 & 22 \\
\hline PrecBBH000119 & 3.0002 & $(0.018,0.29,-0.073)$ & $(-0.055,0.285,-0.076)$ & 0.01600 & 22 \\
\hline PrecBBH000120 & 3.0001 & $(-0.253,0.144,-0.071)$ & $(0.011,-0.286,-0.09)$ & 0.01631 & 22 \\
\hline
\end{tabular}

[1] B. P. Abbott et al. (Virgo, LIGO Scientific Collaborations), Phys. Rev. Lett. 116, 061102 (2016).

[2] J. Aasi et al. (LIGO Scientific Collaboration), Classical Quantum Gravity 32, 115012 (2015).

[3] F. Acernese et al. (Virgo Collaboration), Classical Quantum Gravity 32, 024001 (2015).

[4] B. P. Abbott et al. (LIGO Scientific and Virgo Collaborations), Phys. Rev. X 6, 041015 (2016); 8, 039903(E) (2018).

[5] B. P. Abbott et al. (LIGO Scientific and Virgo Collaborations), Phys. Rev. X 9, 031040 (2019).

[6] B. Zackay, T. Venumadhav, L. Dai, J. Roulet, and M. Zaldarriaga, Phys. Rev. D 100, 023007 (2019).

[7] T. Venumadhav, B. Zackay, J. Roulet, L. Dai, and M. Zaldarriaga, Phys. Rev. D 101, 083030 (2020).

[8] A. H. Nitz, T. Dent, G. S. Davies, S. Kumar, C. D. Capano, I. Harry, S. Mozzon, L. Nuttall, A. Lundgren, and M. Tápai, Astrophys. J. 891, 123 (2020).

[9] R. Abbott et al. (LIGO Scientific and Virgo Collaborations), arXiv:2004.08342.

[10] B. P. Abbott et al. (LIGO Scientific and Virgo Collaborations), Phys. Rev. Lett. 119, 161101 (2017).

[11] B. P. Abbott et al. (LIGO Scientific and Virgo Collaborations), Astrophys. J. Lett. 892, L3 (2020).

[12] B. P. Abbott et al. (LIGO Scientific, Virgo, Fermi GBM, INTEGRAL, IceCube, AstroSat Cadmium Zinc Telluride
Imager Team, IPN, Insight-Hxmt, ANTARES, Swift, AGILE Team, 1M2H Team, Dark Energy Camera GW-EM, DES, DLT40, GRAWITA, Fermi-LAT, ATCA, ASKAP, Las Cumbres Observatory Group, OzGrav, DWF (Deeper Wider Faster Program), AST3, CAASTRO, VINROUGE, MASTER, J-GEM, GROWTH, JAGWAR, CaltechNRAO, TTU-NRAO, NuSTAR, Pan-STARRS, MAXI Team, TZAC Consortium, KU, Nordic Optical Telescope, ePESSTO, GROND, Texas Tech University, SALT Group, TOROS, BOOTES, MWA, CALET, IKIGW Follow-up, H.E.S.S., LOFAR, LWA, HAWC, Pierre Auger, ALMA, Euro VLBI Team, Pi of Sky, Chandra Team at McGill University, DFN, ATLAS Telescopes, High Time Resolution Universe Survey, RIMAS, RATIR, SKA South Africa/MeerKAT Collaborations), Astrophys. J. 848, L12 (2017).

[13] B. P. Abbott et al. (LIGO Scientific, Virgo, Fermi-GBM, and INTEGRAL Collaborations), Astrophys. J. 848, L13 (2017).

[14] B. P. Abbott et al. (KAGRA, LIGO Scientific, and Virgo Collaborations), Living Rev. Relativity 21, 3 (2018).

[15] T. A. Apostolatos, C. Cutler, G. J. Sussman, and K. S. Thorne, Phys. Rev. D 49, 6274 (1994).

[16] L. Blanchet, Living Rev. Relativity 17, 2 (2014).

[17] L. E. Kidder, Phys. Rev. D 52, 821 (1995).

[18] J. D. Schnittman, Phys. Rev. D 70, 124020 (2004). 
[19] M. Kesden, D. Gerosa, R. O’Shaughnessy, E. Berti, and U. Sperhake, Phys. Rev. Lett. 114, 081103 (2015).

[20] D. Gerosa, M. Kesden, U. Sperhake, E. Berti, and R. O’Shaughnessy, Phys. Rev. D 92, 064016 (2015).

[21] A. Buonanno, Y.-b. Chen, and M. Vallisneri, Phys. Rev. D 67, 104025 (2003); 74, 029904(E) (2006).

[22] A. Buonanno, Y. Chen, and T. Damour, Phys. Rev. D 74, 104005 (2006).

[23] P. Schmidt, M. Hannam, S. Husa, and P. Ajith, Phys. Rev. D 84, 024046 (2011).

[24] R. O'Shaughnessy, L. London, J. Healy, and D. Shoemaker, Phys. Rev. D 87, 044038 (2013).

[25] L. Pekowsky, R. O'Shaughnessy, J. Healy, and D. Shoemaker, Phys. Rev. D 88, 024040 (2013).

[26] Y. Pan, A. Buonanno, A. Taracchini, L. E. Kidder, A. H. Mroué, H. P. Pfeiffer, M. A. Scheel, and B. Szilágyi, Phys. Rev. D 89, 084006 (2014).

[27] M. Campanelli, C. O. Lousto, Y. Zlochower, B. Krishnan, and D. Merritt, Phys. Rev. D 75, 064030 (2007).

[28] C. O. Lousto, J. Healy, and H. Nakano, Phys. Rev. D 93, 044031 (2016).

[29] S. Ossokine, M. Boyle, L. E. Kidder, H. P. Pfeiffer, M. A. Scheel, and B. Szilágyi, Phys. Rev. D 92, 104028 (2015).

[30] A. G. M. Lewis, A. Zimmerman, and H. P. Pfeiffer, Classical Quantum Gravity 34, 124001 (2017).

[31] C. Afle et al., Phys. Rev. D 98, 083014 (2018).

[32] A. Taracchini, A. Buonanno, S. A. Hughes, and G. Khanna, Phys. Rev. D 88, 044001 (2013); 88, 109903(E) (2013).

[33] S. Babak, A. Taracchini, and A. Buonanno, Phys. Rev. D 95, 024010 (2017).

[34] M. Hannam, P. Schmidt, A. Bohé, L. Haegel, S. Husa, F. Ohme, G. Pratten, and M. Pürrer, Phys. Rev. Lett. 113, 151101 (2014).

[35] S. Khan, S. Husa, M. Hannam, F. Ohme, M. Pürrer, X. J. Forteza, and A. Bohé, Phys. Rev. D 93, 044007 (2016).

[36] S. Khan, K. Chatziioannou, M. Hannam, and F. Ohme, Phys. Rev. D 100, 024059 (2019).

[37] G. Pratten, S. Husa, C. Garcia-Quiros, M. Colleoni, A. Ramos-Buades, H. Estelles, and R. Jaume, arXiv: 2001.11412.

[38] C. García-Quirós, M. Colleoni, S. Husa, H. Estellés, G. Pratten, A. Ramos-Buades, M. Mateu-Lucena, and R. Jaume, arXiv:2001.10914.

[39] S. Khan, F. Ohme, K. Chatziioannou, and M. Hannam, Phys. Rev. D 101, 024056 (2020).

[40] G. Pratten et al., arXiv:2004.06503.

[41] H. Estellés et al., arXiv:2004.08302.

[42] M. Boyle, R. Owen, and H. P. Pfeiffer, Phys. Rev. D 84, 124011 (2011).

[43] R. O'Shaughnessy, B. Vaishnav, J. Healy, Z. Meeks, and D. Shoemaker, Phys. Rev. D 84, 124002 (2011).

[44] P. Schmidt, M. Hannam, and S. Husa, Phys. Rev. D 86, 104063 (2012).

[45] A. Bohé et al., Phys. Rev. D 95, 044028 (2017).

[46] R. Cotesta, A. Buonanno, A. Bohé, A. Taracchini, I. Hinder, and S. Ossokine, Phys. Rev. D 98, 084028 (2018).

[47] A. H. Mroue et al., Phys. Rev. Lett. 111, 241104 (2013).
[48] T. Chu, H. Fong, P. Kumar, H. P. Pfeiffer, M. Boyle, D. A. Hemberger, L. E. Kidder, M. A. Scheel, and B. Szilagyi, Classical Quantum Gravity 33, 165001 (2016).

[49] E. Barausse, A. Buonanno, S. A. Hughes, G. Khanna, S. O'Sullivan, and Y. Pan, Phys. Rev. D 85, 024046 (2012).

[50] V. Varma, S. E. Field, M. A. Scheel, J. Blackman, L. E. Kidder, and H. P. Pfeiffer, Phys. Rev. D 99, 064045 (2019).

[51] J. Blackman, S. E. Field, M. A. Scheel, C. R. Galley, C. D. Ott, M. Boyle, L. E. Kidder, H. P. Pfeiffer, and B. Szilágyi, Phys. Rev. D 96, 024058 (2017).

[52] V. Varma, S. E. Field, M. A. Scheel, J. Blackman, D. Gerosa, L. C. Stein, L. E. Kidder, and H. P. Pfeiffer, Phys. Rev. Research 1, 033015 (2019).

[53] K. Jani, J. Healy, J. A. Clark, L. London, P. Laguna, and D. Shoemaker, Classical Quantum Gravity 33, 204001 (2016).

[54] J. Healy, C. O. Lousto, Y. Zlochower, and M. Campanelli, Classical Quantum Gravity 34, 224001 (2017).

[55] J. Healy, C. O. Lousto, J. Lange, R. O'Shaughnessy, Y. Zlochower, and M. Campanelli, Phys. Rev. D 100, 024021 (2019).

[56] E. A. Huerta et al., Phys. Rev. D 100, 064003 (2019).

[57] M. Boyle et al., Classical Quantum Gravity 36, 195006 (2019).

[58] I. Hinder, S. Ossokine, H. P. Pfeiffer, and A. Buonanno, Phys. Rev. D 99, 061501 (2019).

[59] B. P. Abbott et al. (Virgo and LIGO Scientific Collaborations), Phys. Rev. X 6, 041014 (2016).

[60] B. P. Abbott et al. (LIGO Scientific and Virgo Collaborations), Phys. Rev. Lett. 118, 221101 (2017); 121, 129901(E) (2018).

[61] M. A. Scheel, M. Giesler, D. A. Hemberger, G. Lovelace, K. Kuper, M. Boyle, B. Szilágyi, and L. E. Kidder, Classical Quantum Gravity 32, 105009 (2015).

[62] G. Lovelace et al., Classical Quantum Gravity 32, 065007 (2015).

[63] B. Szilágyi, J. Blackman, A. Buonanno, A. Taracchini, H. P. Pfeiffer, M. A. Scheel, T. Chu, L. E. Kidder, and Y. Pan, Phys. Rev. Lett. 115, 031102 (2015).

[64] G. Lovelace et al., Classical Quantum Gravity 33, 244002 (2016).

[65] F. Foucart, R. Haas, M. D. Duez, E. O'Connor, C. D. Ott, L. Roberts, L. E. Kidder, J. Lippuner, H. P. Pfeiffer, and M. A. Scheel, Phys. Rev. D 93, 044019 (2016).

[66] R. Haas et al., Phys. Rev. D 93, 124062 (2016).

[67] F. Foucart et al., Phys. Rev. D 99, 044008 (2019).

[68] T. Vincent, F. Foucart, M. D. Duez, R. Haas, L. E. Kidder, H. P. Pfeiffer, and M. A. Scheel, Phys. Rev. D 101, 044053 (2020).

[69] M. Okounkova, M. A. Scheel, and S. A. Teukolsky, Classical Quantum Gravity 36, 054001 (2019).

[70] M. Okounkova, L. C. Stein, M. A. Scheel, and S. A. Teukolsky, Phys. Rev. D 100, 104026 (2019).

[71] M. Okounkova, L. C. Stein, J. Moxon, M. A. Scheel, and S. A. Teukolsky, Phys. Rev. D 101, 104016 (2020).

[72] M. Okounkova, arXiv:2001.03571.

[73] L. Lindblom, M. A. Scheel, L. E. Kidder, R. Owen, and O. Rinne, Classical Quantum Gravity 23, S447 (2006). 
[74] L. Lindblom and B. Szilagyi, Phys. Rev. D 80, 084019 (2009).

[75] B. Szilagyi, L. Lindblom, and M. A. Scheel, Phys. Rev. D 80, 124010 (2009).

[76] D. A. Hemberger, M. A. Scheel, L. E. Kidder, B. Szilágyi, G. Lovelace, N. W. Taylor, and S. A. Teukolsky, Classical Quantum Gravity 30, 115001 (2013).

[77] A. Buonanno, L. E. Kidder, A. H. Mroue, H. P. Pfeiffer, and A. Taracchini, Phys. Rev. D 83, 104034 (2011).

[78] B. S. Sathyaprakash and S. V. Dhurandhar, Phys. Rev. D 44, 3819 (1991).

[79] L. S. Finn and D. F. Chernoff, Phys. Rev. D 47, 2198 (1993).

[80] C. Capano, Y. Pan, and A. Buonanno, Phys. Rev. D 89, 102003 (2014).

[81] L. Barsotti, P. Fritschel, M. Evans, and S. Gras (LIGO Collaboration), Updated advanced ligo sensitivity design curve, LIGO Document T1800044-v, 2018, https://dcc .ligo.org/LIGO-T1800044/public.

[82] M. Boyle, L. E. Kidder, S. Ossokine, and H. P. Pfeiffer, arXiv:1409.4431.

[83] M. Boyle, Phys. Rev. D 87, 104006 (2013).

[84] https://www.lsc-group.phys.uwm.edu/daswg/projects/ lalsuite.html.

[85] A. Taracchini, Y. Pan, A. Buonanno, E. Barausse, M. Boyle, T. Chu, G. Lovelace, H. P. Pfeiffer, and M. A. Scheel, Phys. Rev. D 86, 024011 (2012).

[86] A. Taracchini et al., Phys. Rev. D 89, 061502 (2014).

[87] A. Buonanno and T. Damour, Phys. Rev. D 59, 084006 (1999).

[88] A. Buonanno and T. Damour, Phys. Rev. D 62, 064015 (2000).

[89] T. Damour, P. Jaranowski, and G. Schaefer, Phys. Rev. D 62, 084011 (2000).

[90] T. Damour, Phys. Rev. D 64, 124013 (2001).

[91] E. Barausse, E. Racine, and A. Buonanno, Phys. Rev. D 80, 104025 (2009); 85, 069904(E) (2012).

[92] E. Barausse and A. Buonanno, Phys. Rev. D 81, 084024 (2010).

[93] E. Barausse and A. Buonanno, Phys. Rev. D 84, 104027 (2011).

[94] T. Hinderer and S. Babak, Phys. Rev. D 96, 104048 (2017).

[95] X. Liu, Z. Cao, and L. Shao, Phys. Rev. D 101, 044049 (2020).

[96] D. Chiaramello and A. Nagar, Phys. Rev. D 101, 101501 (R) (2020).

[97] T. Damour and A. Nagar, Phys. Rev. D 76, 064028 (2007).

[98] T. Damour, B. R. Iyer, and A. Nagar, Phys. Rev. D 79, 064004 (2009).

[99] Y. Pan, A. Buonanno, R. Fujita, E. Racine, and H. Tagoshi, Phys. Rev. D 83, 064003 (2011); 87, 109901(E) (2013).

[100] K. G. Arun, A. Buonanno, G. Faye, and E. Ochsner, Phys. Rev. D 79, 104023 (2009); 84, 049901(E) (2011).
[101] C. K. Mishra, A. Kela, K. G. Arun, and G. Faye, Phys. Rev. D 93, 084054 (2016).

[102] S. Bernuzzi, A. Nagar, and A. Zenginoglu, Phys. Rev. D 84, 084026 (2011).

[103] E. Harms, G. Lukes-Gerakopoulos, S. Bernuzzi, and A. Nagar, Phys. Rev. D 93, 044015 (2016).

[104] E. Berti, V. Cardoso, and C. M. Will, Phys. Rev. D 73, 064030 (2006).

[105] S. Ossokine (private communication).

[106] V. Varma, D. Gerosa, L. C. Stein, F. Hébert, and H. Zhang, Phys. Rev. Lett. 122, 011101 (2019).

[107] F. Hofmann, E. Barausse, and L. Rezzolla, Astrophys. J. 825, L19 (2016).

[108] J. M. Bardeen, W. H. Press, and S. A. Teukolsky, Astrophys. J. 178, 347 (1972).

[109] K. Chatziioannou, A. Klein, N. Yunes, and N. Cornish, Phys. Rev. D 95, 104004 (2017).

[110] E. Racine, Phys. Rev. D 78, 044021 (2008).

[111] L. Santamaria et al., Phys. Rev. D 82, 064016 (2010).

[112] P. Schmidt, F. Ohme, and M. Hannam, Phys. Rev. D 91, 024043 (2015).

[113] T. Damour, Phys. Rev. D 81, 024017 (2010).

[114] D. Bini, T. Damour, A. Geralico, C. Kavanagh, and M. van de Meent, Phys. Rev. D 98, 104062 (2018).

[115] A. Antonelli, M. van de Meent, A. Buonanno, J. Steinhoff, and J. Vines, Phys. Rev. D 101, 024024 (2020).

[116] P. Rettegno, F. Martinetti, A. Nagar, D. Bini, G. Riemenschneider, and T. Damour, Phys. Rev. D 101, 104027 (2020).

[117] M. Khalil, J. Steinhoff, J. Vines, and A. Buonanno, Phys. Rev. D 101, 104034 (2020).

[118] A. Nitz, I. Harry, D. Brown, C. M. Biwer, J. Willis, T. D. Canton, C. Capano, L. Pekowsky, T. Dent, A. R. Williamson et al., gwastro/pycbc: РуCBC release v1.15.4 (2020), https://doi.org/10.5281/zenodo.3630601.

[119] R. Smith and G. Ashton, arXiv:1909.11873.

[120] G. Ashton et al., Astrophys. J. Suppl. Ser. 241, 27 (2019).

[121] E. E. Flanagan and S. A. Hughes, Phys. Rev. D 57, 4566 (1998).

[122] L. Lindblom, B. J. Owen, and D. A. Brown, Phys. Rev. D 78, 124020 (2008).

[123] S. T. McWilliams, B. J. Kelly, and J. G. Baker, Phys. Rev. D 82, 024014 (2010).

[124] M. Pürrer and C.-J. Haster, arXiv:1912.10055.

[125] A. Buonanno, G. B. Cook, and F. Pretorius, Phys. Rev. D 75, 124018 (2007).

[126] E. Berti and A. Klein, Phys. Rev. D 90, 064012 (2014).

[127] A. K. Mehta, P. Tiwari, N. K. Johnson-McDaniel, C. K. Mishra, V. Varma, and P. Ajith, Phys. Rev. D 100, 024032 (2019).

[128] B. Gadre et al. (to be published).

[129] A. Nagar and P. Rettegno, Phys. Rev. D 99, 021501 (2019).

[130] https://github.com/moble/GWFrames.

[131] P. Schmidt, I. W. Harry, and H. P. Pfeiffer, arXiv: 1703.01076 . 